\title{
The Rigidity of Infinite Graphs
}

\author{
Derek Kitson $^{1}$ (D) S Stephen C. Power ${ }^{1}$
}

Received: 6 December 2016 / Revised: 27 February 2018 / Accepted: 23 March 2018 /

Published online: 11 April 2018

(C) The Author(s) 2018

\begin{abstract}
A rigidity theory is developed for countably infinite simple graphs in $\mathbb{R}^{d}$. Generalisations are obtained for the Laman combinatorial characterisation of generic infinitesimal rigidity for finite graphs in $\mathbb{R}^{2}$ and Tay's multi-graph characterisation of generic infinitesimal rigidity for finite body-bar frameworks in $\mathbb{R}^{d}$. Analogous results are obtained for the classical non-Euclidean $\ell^{q}$ norms.
\end{abstract}

Keywords Infinite graphs · Infinitesimal rigidity $\cdot$ Combinatorial rigidity

Mathematics Subject Classification 52C25 - 05C63

\section{Introduction}

In 1864 James Clerk Maxwell [25] initiated a combinatorial trend in the rigidity theory of finite bar-joint frameworks in Euclidean space. In two dimensions this amounted to the observation that the underlying structure graph $G=(V, E)$ must satisfy the simple counting rule $|E| \geq 2|V|-3$. For minimal rigidity, in which any bar removal renders the framework flexible, equality must hold together with the inequalities $|E(H)| \leq$

\section{Editor in Charge: János Pach}

Supported by the Engineering and Physical Sciences Research Council [grant number EP/J008648/1].

Derek Kitson

d.kitson@lancaster.ac.uk

Stephen C. Power

s.power@lancaster.ac.uk

1 The Department of Mathematics and Statistics, Lancaster University, Lancaster LA1 4YF, UK 
$2|V(H)|-3$ for subgraphs $H$ with at least two vertices. The fundamental result that these two necessary conditions are also sufficient for the minimal rigidity of a generic framework was obtained by Laman in 1970 and this has given impetus to the development of matroid theory techniques. While corresponding counting rules are necessary in three dimensions they fail to be sufficient and a purely combinatorial characterisation of generic rigidity is not available. On the other hand many specific families of finite graphs are known to be generically rigid, such as the edge graphs of triangle-faced convex polyhedra in three dimensions and the graphs associated with finite triangulations of general surfaces. See, for example, Alexandrov [1], Fogelsanger [9], Gluck [10], Kann [16] and Whiteley [42,43].

A finite simple graph $G$ is said to be generically $d$-rigid, or simply $d$-rigid, if its realisation as some generic bar-joint framework in the Euclidean space $\mathbb{R}^{d}$ is infinitesimally rigid. Here generic refers to the algebraic independence of the set of coordinates of the vertices and infinitesimal rigidity in this case is equivalent to continuous (non-trivial finite motion) rigidity (Asimow and Roth [2,3]). The rigidity analysis of bar-joint frameworks and related frameworks, such as body-bar frameworks and body-hinge frameworks, continues to be a focus of investigation, both in the generic case and in the presence of symmetry. For example Katoh and Tanigawa [18] have resolved the molecular conjecture for generic structures, while Schulze [38] has obtained variants of Laman's theorem for semi-generic symmetric bar-joint frameworks. In the case of infinite frameworks however developments have centred mainly on periodic frameworks and the infinitesimal and finite motions which retain some form of periodicity. Indeed, periodicity hypotheses lead to configuration spaces that are real algebraic varieties and so to methods from multi-linear algebra and finite combinatorics. See, for example, Borcea and Streinu [4], Connelly et al. [8], Malestein and Theran [24], Owen and Power [32] and Ross et al. [37]. Periodic rigidity, broadly interpreted, is also significant in a range of applied settings, such as the mathematical analysis of rigid unit modes in crystals, as indicated in Power [34] and Wegner [41], for example.

In the development below we consider general countable simple graphs and the flexibility and rigidity of their placements in the Euclidean spaces $\mathbb{R}^{d}$ and in the non-Euclidean spaces $\left(\mathbb{R}^{d},\|\cdot\|_{q}\right)$ for the classical $\ell^{q}$ norms, for $1<q<\infty$. The constraint conditions for the non-Euclidean $\ell^{q}$ norms are no longer given by polynomial equations and so we adapt the Asimow-Roth notion of a regular framework to obtain the appropriate form of a generic framework. This strand of norm constraint rigidity theory for finite frameworks was initiated in [20] for $\ell^{q}$ norms. It was further developed in [19] for polyhedral norms and in [22] for general norms. We continue this development in Theorem 5.5 where we generalise Tay's multi-graph characterisation [40] of generically rigid finite body-bar frameworks in $\mathbb{R}^{d}$ to the non-Euclidean $\ell^{q}$ norms. As well as being a natural problem, one of the original motivations for considering rigidity with respect to a different norm was based on similarities which arose with the combinatorial methodologies used for surface-constrained frameworks $[28,29]$ and the potential for cross-fertilization between these topics. Subsequently, norm based rigidity has gained interest in relation to metric embeddability [39].

Our first main result is Theorem 1.1 in which we determine the simple countable graphs which are locally generically rigid for $\left(\mathbb{R}^{2},\|\cdot\|_{q}\right)$, for $1<q<\infty$. This is 
a generalisation of Laman's theorem (and its non-Euclidean analogue) to countable graphs.

Theorem 1.1 Let $G$ be a countable simple graph.

(A) The following statements are equivalent.

(i) $G$ is rigid in $\left(\mathbb{R}^{2},\|\cdot\|_{2}\right)$.

(ii) $G$ contains a (2,3)-tight vertex-complete tower.

(B) If $q \in(1,2) \cup(2, \infty)$ then the following statements are equivalent.

(i) $G$ is rigid in $\left(\mathbb{R}^{2},\|\cdot\|_{q}\right)$.

(ii) $G$ contains a $(2,2)$-tight vertex-complete tower.

We also see that these graphs are necessarily sequentially rigid in the sense of containing a spanning subgraph which is a union of finite graphs, each of which is infinitesimally rigid. This is the strongest form of infinitesimal rigidity and its equivalence with infinitesimal rigidity is particular to two dimensions; an infinite chain of double banana graphs shows that the corresponding equivalence fails to hold in higher dimensions (see Fig. 2).

These results rest in part on a general characterisation of infinitesimal rigidity in terms of what we refer to as the relative rigidity of a finite graph $G_{1}$ with respect to a containing finite graph $G_{2}$. Specifically, for all dimensions and norms we show that a countable simple graph $G$ is infinitesimally rigid if and only if there is a subgraph inclusion tower

$$
G_{1} \subseteq G_{2} \subseteq G_{3} \subseteq \cdots
$$

which is vertex spanning and in which for each $k$ the graph $G_{k}$ is relatively infinitesimally rigid in $G_{k+1}$ (Theorem 3.14). This relative rigidity principle seems to be generally useful in the characterisation of generic rigidity for infinite geometric frameworks in a variety of contexts. In our second main result, in Sect. 5, we illustrate this by generalising Tay's theorem to infinite locally generic body-bar frameworks (Theorem 1.2). That is we characterise such frameworks combinatorially in terms of their associated countably infinite multi-graphs $G_{b}$.

Theorem 1.2 Let $G$ be a countable multi-body graph for $\left(\mathbb{R}^{d},\|\cdot\|_{q}\right)$ where $q \in$ $(1, \infty)$.

(A) The following statements are equivalent.

(i) $G$ is rigid in $\left(\mathbb{R}^{d},\|\cdot\|_{2}\right)$.

(ii) $G_{b}$ has a $\left(\frac{d(d+1)}{2}, \frac{d(d+1)}{2}\right)$-tight vertex-complete tower.

(B) If $q \in(1,2) \cup(2, \infty)$ then the following statements are equivalent.

(i) $G$ is rigid in $\left(\mathbb{R}^{d},\|\cdot\|_{q}\right)$.

(ii) $G_{b}$ has a $(d, d)$-tight vertex-complete tower. 
We comment on further directions and related problems at the end of Sect. 3. Accounts of the foundations of geometric rigidity theory are given in Alexandrov [1], Graver [12], Graver et al. [13] and Whiteley [45]. Also [13] has a comprehensive guide to the literature up to 1993. The influential papers of Asimow and Roth introduced regular frameworks as a more appropriate form of genericity and in Definition 2.6 we have followed Graver in requiring that all frameworks supported by vertices of $G$ should be regular.

\section{Preliminaries}

In this section we state necessary definitions for finite and countably infinite graphs and we review the necessary background on the rigidity of finite graphs in $\mathbb{R}^{d}$ with respect to the classical $\ell^{q}$ norms.

\subsection{Continuous and Infinitesimal Rigidity}

A bar-joint framework in a normed vector space $(X,\|\cdot\|)$ is a pair $(G, p)$ consisting of a simple graph $G=(V(G), E(G))$ and a mapping $p: V(G) \rightarrow X, v \mapsto p_{v}$, with the property that $p_{v} \neq p_{w}$ whenever $v w \in E(G)$. Unless otherwise stated, the vertex set $V(G)$ is allowed to be either finite or countably infinite. We call $p$ a placement of $G$ in $X$ and the collection of all placements of $G$ in $X$ will be denoted by $P(G, X)$ or simply $P(G)$ when the context is clear. If $H$ is a subgraph of $G$ then the bar-joint framework $(H, p)$ obtained by restricting $p$ to $V(H)$ is called a subframework of $(G, p)$.

Definition 2.1 A continuous flex of $(G, p)$ is a family of continuous paths

$$
\alpha_{v}:[-1,1] \rightarrow X, \quad v \in V(G),
$$

such that $\alpha_{v}(0)=p_{v}$ for all $v \in V(G)$ and $\left\|\alpha_{v}(t)-\alpha_{w}(t)\right\|=\left\|p_{v}-p_{w}\right\|$ for all $t \in[-1,1]$ and all $v w \in E(G)$.

A continuous flex is regarded as trivial if it results from a continuous isometric motion of the ambient space. Formally, a continuous rigid motion of $(X,\|\cdot\|)$ is a mapping $\Gamma(x, t): X \times[-1,1] \rightarrow X$ which is isometric in the variable $x$ and continuous in the variable $t$ with $\Gamma(x, 0)=x$ for all $x \in X$. Every continuous rigid motion gives rise to a continuous flex of $(G, p)$ by setting $\alpha_{v}:[-1,1] \rightarrow X, t \mapsto \Gamma\left(p_{v}, t\right)$, for each $v \in V(G)$. A continuous flex of $(G, p)$ is trivial if it can be derived from a continuous rigid motion in this way. If every continuous flex of $(G, p)$ is trivial then we say that $(G, p)$ is continuously rigid, otherwise we say that $(G, p)$ is continuously flexible.

Definition 2.2 An infinitesimal flex of $(G, p)$ is a mapping $u: V(G) \rightarrow X, v \mapsto u_{v}$, which satisfies

$$
\left\|\left(p_{v}+t u_{v}\right)-\left(p_{w}+t u_{w}\right)\right\|-\left\|p_{v}-p_{w}\right\|=o(t), \quad \text { as } t \rightarrow 0
$$

for each edge $v w \in E(G)$. 
We will denote the vector space of infinitesimal flexes of $(G, p)$ by $\mathcal{F}(G, p)$. An infinitesimal rigid motion of $(X,\|\cdot\|)$ is a mapping $\gamma: X \rightarrow X$ derived from a continuous rigid motion $\Gamma$ by the formula $\gamma(x)=\left.\frac{d}{d t} \Gamma(x, t)\right|_{t=0}$ for all $x \in X$. The vector space of all infinitesimal rigid motions of $(X,\|\cdot\|)$ is denoted $\mathcal{T}(X)$. Every infinitesimal rigid motion $\gamma \in \mathcal{T}(X)$ gives rise to an infinitesimal flex of $(G, p)$ by setting $u_{v}=\gamma\left(p_{v}\right)$ for all $v \in V(G)$. We regard such infinitesimal flexes as trivial and the collection of all trivial infinitesimal flexes of $(G, p)$ is a vector subspace of $\mathcal{F}(G, p)$ which we denote by $\mathcal{T}(G, p)$. The infinitesimal flexibility dimension of $(G, p)$ is the vector space dimension of the quotient space,

$$
\operatorname{dim}_{\mathrm{fl}}(G, p):=\operatorname{dim} \mathcal{F}(G, p) / \mathcal{T}(G, p)
$$

If $\mathcal{T}(G, p)$ is a proper subspace then $(G, p)$ is said to be an infinitesimally flexible bar-joint framework. Otherwise, we say that $(G, p)$ is infinitesimally rigid and we call $p$ an infinitesimally rigid placement of $G$. A bar-joint framework $(G, p)$ is minimally infinitesimally rigid if it is infinitesimally rigid and removing any edge results in a subframework which is infinitesimally flexible.

We will consider the rigidity properties of bar-joint frameworks in $\mathbb{R}^{d}$ with respect to the family $\left\{\|\cdot\|_{q}: q \in(1, \infty)\right\}$ of $\ell^{q}$ norms,

$$
\|\cdot\|_{q}: \mathbb{R}^{d} \rightarrow \mathbb{R}, \quad\left\|\left(x_{1}, \ldots, x_{d}\right)\right\|_{q}=\left(\sum_{i=1}^{d}\left|x_{i}\right|^{q}\right)^{\frac{1}{q}}
$$

We use a subscript $q$ to indicate the $\ell^{q}$ norm when referring to the collection of infinitesimal rigid motions $\mathcal{T}_{q}\left(\mathbb{R}^{d}\right)$, the infinitesimal flexes $\mathcal{F}_{q}(G, p)$, and trivial infinitesimal flexes $\mathcal{T}_{q}(G, p)$ of a bar-joint framework. In the Euclidean setting $q=2$ it is well-known that the space of infinitesimal rigid motions $\mathcal{T}_{2}\left(\mathbb{R}^{d}\right)$ has dimension $\frac{d(d+1)}{2}$. In the non-Euclidean setting $q \neq 2$ the infinitesimal rigid motions are precisely the constant mappings and so $\mathcal{T}_{q}\left(\mathbb{R}^{d}\right)$ is $d$-dimensional (see [20, Lem. 2.2]).

Proposition 2.3 Let $(G, p)$ be a bar-joint framework in $\left(\mathbb{R}^{d},\|\cdot\|_{q}\right)$ where $q \in(1, \infty)$. Then a mapping $u: V(G) \rightarrow \mathbb{R}^{d}$ is an infinitesimal flex of $(G, p)$ if and only if

$$
\sum_{i=1}^{d} \operatorname{sgn}\left(p_{v, i}-p_{w, i}\right)\left|p_{v, i}-p_{w, i}\right|^{q-1}\left(u_{v, i}-u_{w, i}\right)=0
$$

for each edge $v w \in E(G)$.

Proof See proof of [20, Prop. 3.1].

If $G$ is a finite graph then the system of linear equations in Proposition 2.3 can be expressed as a matrix equation $R_{q}(G, p) u=0$ where $R_{q}(G, p)$ is an $|E(G)| \times$ $d|V(G)|$ matrix called the rigidity matrix for $(G, p)$. The rows of $R_{q}(G, p)$ are indexed by the edges of $G$ and the columns are indexed by the $d$ coordinates of $p_{v}$ for each vertex $v \in V(G)$. The row entries for a particular edge $v w \in E(G)$ are 


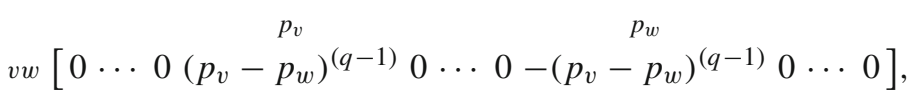

where we use the notation $x^{(q)}=\left(\operatorname{sgn}\left(x_{1}\right)\left|x_{1}\right|^{q}, \ldots, \operatorname{sgn}\left(x_{d}\right)\left|x_{d}\right|^{q}\right)$. Evidently we have $\mathcal{F}_{q}(G, p) \cong \operatorname{ker} R_{q}(G, p)$ for all $q \in(1, \infty)$ and it immediately follows that

$$
\operatorname{rank} R_{q}(G, p) \leq d|V(G)|-\operatorname{dim} \mathcal{T}_{q}(G, p)
$$

with equality if and only if $(G, p)$ is infinitesimally rigid.

Definition 2.4 A finite bar-joint framework $(G, p)$ is regular in $\left(\mathbb{R}^{d},\|\cdot\|_{q}\right)$ if the function

$$
P\left(G, \mathbb{R}^{d}\right) \rightarrow \mathbb{R}, \quad x \mapsto \operatorname{rank} R_{q}(G, x)
$$

achieves its maximum value at $p$.

The equivalence of continuous and infinitesimal rigidity for regular finite bar-joint frameworks in Euclidean space was established by Asimow and Roth [2,3]. In [21] this result is extended to finite bar-joint frameworks in the non-Euclidean spaces $\left(\mathbb{R}^{d},\|\cdot\|_{q}\right)$ for $q \in(1, \infty)$.

Theorem 2.5 (Asimow-Roth [2,3]) If $(G, p)$ is a finite bar-joint framework in Euclidean space $\left(\mathbb{R}^{d},\|\cdot\|_{2}\right)$ then the following statements are equivalent.

(i) $(G, p)$ is continuously rigid and regular.

(ii) $(G, p)$ is infinitesimally rigid.

We now formalise our meaning of a generic finite bar-joint framework in $\left(\mathbb{R}^{d},\|\cdot\|_{q}\right)$ for $q \in(1, \infty)$. The complete graph on the vertices $V(G)$ will be denoted $K_{V(G)}$.

Definition 2.6 A finite bar-joint framework $(G, p)$ is generic in $\left(\mathbb{R}^{d},\|\cdot\|_{q}\right)$ if $p \in$ $P\left(K_{V(G)}, \mathbb{R}^{d}\right)$ and every subframework of $\left(K_{V(G)}, p\right)$ is regular.

If $(G, p)$ is a finite bar-joint framework then $p$ will frequently be identified with a vector $\left(p_{v_{1}}, p_{v_{2}}, \ldots, p_{v_{n}}\right) \in \mathbb{R}^{d|V(G)|}$ with respect to some fixed ordering of the vertices $V(G)=\left\{v_{1}, v_{2}, \ldots, v_{n}\right\}$. In particular, the collection of all generic placements of $G$ in $\left(\mathbb{R}^{d},\|\cdot\|_{q}\right)$ is identified with a subset of $\mathbb{R}^{d|V(G)|}$.

Lemma 2.7 Let $G$ be a finite simple graph and let $q \in(1, \infty)$. Then the set of generic placements of $G$ in $\left(\mathbb{R}^{d},\|\cdot\|_{q}\right)$ is an open and dense subset of $\mathbb{R}^{d|V(G)|}$.

Proof The set of regular placements of $G$ is an open set since the rank function is lower semi-continuous and the matrix-valued function $x \mapsto R_{q}(G, x)$ is continuous. Let $\mathcal{V}_{n r}(G)$ denote the set of all non-regular placements of $G$ and let $\mathcal{V}(G)$ be the variety

$$
\mathcal{V}(G):=\left\{x \in \mathbb{R}^{d|V(G)|}: \prod_{v w \in E(G)} \prod_{i=1}^{d}\left(x_{v, i}-x_{w, i}\right)=0\right\} .
$$


If $p \in \mathcal{V}_{n r}(G) \backslash \mathcal{V}(G)$ then there exists a neighbourhood $U$ of $p$ such that

$$
\mathcal{V}_{n r}(G) \cap U=\left\{x \in U: \phi_{1}(x)=\cdots=\phi_{m}(x)=0\right\},
$$

where $\phi_{1}(x), \ldots, \phi_{m}(x)$ are the minors of $R_{q}(G, x)$ which correspond to its largest square submatrices. The entries of $R_{q}(G, x)$ when viewed as functions of $x$ are real analytic at all points in the complement of $\mathcal{V}(G)$ and so in particular we may assume that $\phi_{1}, \ldots, \phi_{m}$ are real analytic on $U$. Thus $\mathcal{V}_{n r}(G) \backslash \mathcal{V}(G)$ is a real analytic set in $\mathbb{R}^{d|V(G)|}$ and so the set of regular placements of $G$ is a dense subset of $\mathbb{R}^{d|V(G)|}$. Finally, the set of generic placements of $G$ is obtained as a finite intersection of open and dense sets.

Note that the infinitesimal flexibility dimension $\operatorname{dim}_{\mathrm{fl}}(G, p)$ is constant on the set of generic placements of $G$ in $\left(\mathbb{R}^{d},\|\cdot\|_{q}\right)$. Also, if $G$ has a (minimally) infinitesimally rigid placement then all generic placements of $G$ are (minimally) infinitesimally rigid in $\left(\mathbb{R}^{d},\|\cdot\|_{q}\right)$.

Definition 2.8 Let $G$ be a finite simple graph.

1. The infinitesimal flexibility dimension of $G$ in $\left(\mathbb{R}^{d},\|\cdot\|_{q}\right)$ is

$$
\operatorname{dim}_{\mathrm{fl}}(G):=\operatorname{dim}_{d, q}(G):=\operatorname{dim}_{\mathrm{fl}}(G, p)=\operatorname{dim} \mathcal{F}_{q}(G, p) / \mathcal{T}_{q}(G, p) .
$$

where $p$ is any generic placement of $G$.

2. $G$ is (minimally) rigid in $\left(\mathbb{R}^{d},\|\cdot\|_{q}\right)$ if the generic placements of $G$ are (minimally) infinitesimally rigid.

One can readily verify that the complete graph $K_{d+1}$ on $d+1$ vertices satisfies $\operatorname{dim}_{d, 2}\left(K_{d+1}\right)=0$ and that $K_{d+1}$ is minimally rigid for $\mathbb{R}^{d}$ with the Euclidean norm. Also, in $d$ dimensions we have $\operatorname{dim}_{d, q}\left(K_{2 d}\right)=0$, with minimal rigidity, for each of the non-Euclidean $q$-norms.

\subsection{Sparsity and Rigidity}

We recall the following classes of multi-graphs.

Definition 2.9 Let $k, l \in \mathbb{N}$ with either $(k, l)=(2,3)$ or $k=l$. A multi-graph $G$ is

1. $(k, l)$-sparse if $|E(H)| \leq k|V(H)|-l$ for each subgraph $H$ of $G$ which contains at least two vertices.

2. $(k, l)$-tight if it is $(k, l)$-sparse and $|E(G)|=k|V(G)|-l$.

Our main interests are in the classes of simple (2, 2)-sparse and (2, 3)-sparse graphs and the class of $(k, k)$-sparse multi-graphs for $k \geq 2$.

Example 2.10 The complete graph $K_{n}$ is $(k, k)$-sparse for $1 \leq n \leq 2 k,(k, k)$-tight for $n \in\{1,2 k\}$ and fails to be $(k, k)$-sparse for $n>2 k$. Also, $K_{2}$ and $K_{3}$ are (2, 3)-tight while $K_{n}$ fails to be $(2,3)$-sparse for $n \geq 4$. 
Laman's theorem [23] provides a combinatorial characterisation of the class of finite simple graphs which are rigid in the Euclidean plane and can be restated as follows.

Theorem 2.11 (Laman [23]) If G is a finite simple graph then the following statements are equivalent.

(i) $G$ is rigid in $\left(\mathbb{R}^{2},\|\cdot\|_{2}\right)$.

(ii) G contains a (2, 3)-tight spanning subgraph.

In particular, a generic bar-joint framework $(G, p)$ is minimally infinitesimally rigid in $\left(\mathbb{R}^{2},\|\cdot\|_{2}\right)$ if and only if $G$ is $(2,3)$-tight. In [20] the following analogue of Laman's theorem was obtained for the non-Euclidean $\ell^{q}$ norms.

Theorem 2.12 [20] If $G$ is a finite simple graph and $q \in(1,2) \cup(2, \infty)$ then the following statements are equivalent.

(i) $G$ is rigid in $\left(\mathbb{R}^{2},\|\cdot\|_{q}\right)$.

(ii) $G$ contains a $(2,2)-t i g h t$ spanning subgraph.

\section{Rigidity of Countable Graphs}

In this section we establish the general principle that infinitesimal rigidity is equivalent to local relative rigidity in the sense that every finite subframework is rigid relative to some finite containing superframework (Theorem 3.14). Following this we prove Theorem 1.1 which is the generalised Laman theorem. The rigidity of general infinite graphs as bar-joint frameworks was considered first in Owen and Power [30,32] and part (A) of Theorem 1.1 answers a question posed in [32].

\subsection{Sparsity Lemmas}

We first obtain characterisations of $(k, l)$-tightness which are needed for the construction of inclusion chains of rigid graphs.

Lemma 3.1 Let $G$ be a $(k, l)$-sparse multi-graph containing vertices $v, w \in V(G)$ with $v w \notin E(G)$ and let $G^{\prime}=G \cup\{v w\}$. Then exactly one of the following conditions must hold.

(i) $G^{\prime}$ is $(k, l)$-sparse, or,

(ii) there exists a $(k, l)$-tight subgraph of $G$ which contains both $v$ and $w$.

Proof If $G^{\prime}$ is not $(k, l)$-sparse then there exists a subgraph $H^{\prime}$ of $G^{\prime}$ which fails the sparsity count. Now $H^{\prime} \backslash\{v w\}$ is a $(k, l)$-tight subgraph of $G$ which contains both $v$ and $w$. Conversely, if $H$ is a $(k, l)$-tight subgraph of $G$ which contains both $v$ and $w$ then $H \cup\{v w\}$ is a subgraph of $G^{\prime}$ which fails the sparsity count.

Lemma 3.2 Let $G$ be a $(k, l)$-sparse multi-graph. Suppose that one of the following conditions holds. 
(a) $k=2, l=3$ and $G$ contains at least two vertices, or,

(b) $k=l$ and $G$ contains at least $2 k$ vertices.

Then $G$ is a spanning subgraph of a $(k, l)$-tight graph $G^{\prime}$ obtained by adjoining edges of the form $v w$ to $E(G)$ where $v$ and $w$ are distinct vertices of $V(G)$.

Proof Let $K$ be the complete graph on the vertices of $G$. The collection of edge sets of the $(k, l)$-sparse subgraphs of $K$ form the independent sets of a matroid. Moreover, the edge sets of the $(k, l)$-tight subgraphs of $K$ are the base elements of this matroid. In case (a), this is well-known and a consequence of Laman's theorem, while case (b) follows from Nash-Williams characterisation [26] of these graphs as those where the edge set is the disjoint union of $k$ spanning forests. Each independent set in a matroid extends to a base element and so, in particular, the edge set of $G$ extends to the edge set of a $(k, l)$-tight graph $G^{\prime}$ on the same vertex set.

\subsection{Relative Infinitesimal Rigidity}

We first prove that in two dimensional $\ell^{q}$ spaces relative infinitesimal rigidity is equivalent to the existence of a rigid containing framework.

Definition 3.3 Let $(G, p)$ be a bar-joint framework in a normed space $(X,\|\cdot\|)$.

1. A subframework $(H, p)$ is relatively infinitesimally rigid in $(G, p)$ if there is no non-trivial infinitesimal flex of $(H, p)$ which extends to an infinitesimal flex of $(G, p)$.

2. A subframework $(H, p)$ has an infinitesimally rigid container in $(G, p)$ if there exists an infinitesimally rigid subframework of $(G, p)$ which contains $(H, p)$ as a subframework.

If the complete bar-joint framework $\left(K_{V(H)}, p\right)$ is infinitesimally rigid in $(X,\|\cdot\|)$ then relative infinitesimal rigidity is characterised by the property

$$
\mathcal{F}(G, p)=\mathcal{F}\left(G \cup K_{V(H)}, p\right)
$$

It follows that relative infinitesimal rigidity is a generic property for bar-joint frameworks in $\left(\mathbb{R}^{d},\|\cdot\|_{q}\right)$ for all $q \in(1, \infty)$ since if $p$ and $\tilde{p}$ are two generic placements of $G$ then

$$
\mathcal{F}_{q}(G, \tilde{p}) \cong \mathcal{F}_{q}(G, p)=\mathcal{F}_{q}\left(G \cup K_{V(H)}, p\right) \cong \mathcal{F}_{q}\left(G \cup K_{V(H)}, \tilde{p}\right)
$$

To ensure that $\left(K_{V(H)}, p\right)$ is infinitesimally rigid in the Euclidean case we require that $H$ contains at least $d+1$ vertices while in the non-Euclidean cases $H$ must contain at least $2 d$ vertices. We will say that a subgraph $H$ is relatively rigid in $G$ with respect to $\left(\mathbb{R}^{d},\|\cdot\|_{q}\right)$ if the subframework $(H, p)$ is relatively infinitesimally rigid in $(G, p)$ for some (and hence every) generic placement of $G$. Note that the existence of an infinitesimally rigid container is also a generic property for bar-joint frameworks in $\left(\mathbb{R}^{d},\|\cdot\|_{q}\right)$. We will say that a subgraph $H$ has a rigid container in $G$ with respect to $\left(\mathbb{R}^{d},\|\cdot\|_{q}\right)$ if there exists a rigid subgraph of $G$ which contains $H$. 
Fig. 1 An example of a relatively rigid subgraph in the Euclidean space $\mathbb{R}^{3}$ which does not have a rigid container

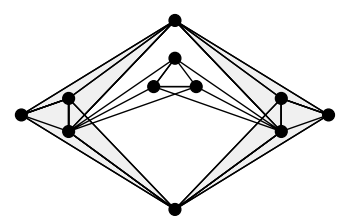

If $(H, p)$ has an infinitesimally rigid container in $(G, p)$ then $(H, p)$ is relatively infinitesimally rigid in $(G, p)$. The converse statement is not true in general as the following example shows.

Example 3.4 Figure 1 illustrates a generic bar-joint framework $(G, p)$ in $\left(\mathbb{R}^{3},\|\cdot\|_{2}\right)$ with subframework $(H, p)$ indicated by the shaded region. Note that $(H, p)$ is relatively infinitesimally rigid in $(G, p)$ but does not have an infinitesimally rigid container in $(G, p)$.

In the following we will say that a finite simple graph $G$ is independent in $\left(\mathbb{R}^{d},\|\cdot\|_{q}\right)$ if the rigidity matrix $R_{q}(G, p)$ is independent for some (and hence every) generic placement $p: V(G) \rightarrow \mathbb{R}^{d}$.

Proposition 3.5 Let $G$ be a finite simple graph and let $q \in(1, \infty)$. Suppose that one of the following conditions holds.

(a) $q=2, l=3$ and $G$ contains at least two vertices, or,

(b) $q \neq 2, l=2$ and $G$ contains at least four vertices.

Then the following statements are equivalent.

(i) $G$ is independent in $\left(\mathbb{R}^{2},\|\cdot\|_{q}\right)$.

(ii) $G$ is $(2, l)$-sparse.

Proof Let $p: V(G) \rightarrow \mathbb{R}^{2}$ be a generic placement of $G$ in $\left(\mathbb{R}^{2},\|\cdot\|_{q}\right)$. If $G$ is independent and $H$ is a subgraph of $G$ then $|E(H)|=\operatorname{rank} R_{q}(H, p) \leq 2|V(H)|-l$. We conclude that $G$ is $(2, l)$-sparse.

Conversely, if $G$ is $(2, l)$-sparse then, by Lemma 3.2, $G$ is a subgraph of some (2,l)-tight graph $G^{\prime}$ with $V(G)=V\left(G^{\prime}\right)$. By Laman's theorem and its analogue for the non-Euclidean case (Theorems $2.11,2.12),\left(G^{\prime}, p\right)$ is minimally infinitesimally rigid and so $G$ is independent.

We now show that relative infinitesimal rigidity does imply the existence of an infinitesimally rigid container for generic bar-joint frameworks in $\left(\mathbb{R}^{2},\|\cdot\|_{q}\right)$ for all $q \in(1, \infty)$.

Theorem 3.6 Let $G$ be a finite simple graph and let $H$ be a subgraph of $G$. Suppose that $q \in(1, \infty)$ and that one of the following conditions holds.

(a) $q=2$ and $H$ contains at least two vertices, or,

(b) $q \neq 2$ and $H$ contains at least four vertices.

Then the following statements are equivalent. 
(i) $H$ is relatively rigid in $G$ with respect to $\left(\mathbb{R}^{2},\|\cdot\|_{q}\right)$.

(ii) $H$ has a rigid container in $G$ with respect to $\left(\mathbb{R}^{2},\|\cdot\|_{q}\right)$.

Proof (i) $\Rightarrow$ (ii) Consider first the case when $G$ is independent with respect to $\left(\mathbb{R}^{2},\|\cdot\|_{q}\right)$. By Proposition $3.5, G$ is $(2, l)$-sparse (where $l=3$ when $q=2$ and $l=2$ when $q \neq 2$ ). Since $K_{V(H)}$ is rigid in $\left(\mathbb{R}^{2},\|\cdot\|_{q}\right)$ the relative rigidity property implies that

$$
\mathcal{F}_{q}(G, p)=\mathcal{F}_{q}\left(G \cup K_{V(H)}, p\right)
$$

for every generic placement $p \in \mathcal{P}(G)$. It follows that if $v, w \in V(H)$ and $v w \notin E(G)$ then $G \cup\{v w\}$ is dependent. By Proposition 3.5, $G \cup\{v w\}$ is not $(2, l)$-sparse. Thus by Lemma 3.1 there exists a $(2, l)$-tight subgraph $H_{v, w}$ of $G$ with $v, w \in V\left(H_{v, w}\right)$. By Theorems 2.11 and 2.12, $H_{v, w}$ is rigid in $\left(\mathbb{R}^{2},\|\cdot\|_{q}\right)$. Let $H^{\prime}$ be the subgraph of $G$ which consists of $H$ and the subgraphs $H_{v, w}$. Then $H^{\prime}$ is rigid in $\left(\mathbb{R}^{2},\|\cdot\|_{q}\right)$ and so $H^{\prime}$ is a rigid container for $H$ in $G$.

If $G$ is dependent then let $p: V(G) \rightarrow \mathbb{R}^{2}$ be a generic placement of $G$. There exists an edge $v w \in E(G)$ such that

$$
\operatorname{ker} R_{q}(G, p)=\operatorname{ker} R_{q}(G \backslash\{v w\}, p) \text {. }
$$

Let $G_{1}=G \backslash\{v w\}$ and $H_{1}=H \backslash\{v w\}$ and note that $H_{1}$ is relatively rigid in $G_{1}$. Continuing to remove edges in this way we arrive after finitely many iterations at subgraphs $H_{n}$ and $G_{n}$ such that $V\left(H_{n}\right)=V(H), H_{n}$ is relatively rigid in $G_{n}$ and $G_{n}$ is independent. By the above argument there exists a rigid container $H_{n}^{\prime}$ for $H_{n}$ in $G_{n}$. Now $H^{\prime}=H_{n}^{\prime} \cup H$ is a rigid container for $H$ in $G$.

(ii) $\Rightarrow$ (i) Let $H^{\prime}$ be a rigid container for $H$ in $G$ and let $p: V(G) \rightarrow \mathbb{R}^{2}$ be a generic placement of $G$ in $\left(\mathbb{R}^{2},\|\cdot\|_{q}\right)$. Then no non-trivial infinitesimal flex of $(H, p)$ can be extended to an infinitesimal flex of $\left(H^{\prime}, p\right)$ and so the result follows.

Remark 3.7 In their analysis of globally linked pairs of vertices in rigid frameworks Jackson et al. [15] remark that it follows from the characterisation of independent sets for the rigidity matroid for the Euclidean plane that linked vertices $\left\{v_{1}, v_{2}\right\}$ must lie in the same rigid component. (See also [14].) This assertion is essentially equivalent to part (a) of Theorem 3.6. The terminology here is that a pair of vertices $\left\{v_{1}, v_{2}\right\}$ in a graph $G$ is linked in $(G, p)$ if there exists an $\epsilon>0$ such that if $q \in P(G)$ is another placement of $G$ with $\left\|q_{v}-q_{w}\right\|_{2}=\left\|p_{v}-p_{w}\right\|_{2}$ for all $v w \in E(G)$ and $\left\|q_{v}-p_{v}\right\|_{2}<\epsilon$ for all $v \in V(G)$ then $\left\|q_{v_{1}}-q_{v_{2}}\right\|_{2}=\left\|p_{v_{1}}-p_{v_{2}}\right\|_{2}$. It can be shown that this is a generic property and that a subgraph $H \subseteq G$ is relatively rigid in $G$ if and only if for a generic placement $(G, p)$ each pair of vertices in $H$ is linked in $(G, p)$.

\subsection{Flex Cancellation and Relatively Rigid Towers}

A tower of bar-joint frameworks in a normed vector space $(X,\|\cdot\|)$ is a sequence $\left\{\left(G_{k}, p_{k}\right): k \in \mathbb{N}\right\}$ of finite bar-joint frameworks in $(X,\|\cdot\|)$ such that $\left(G_{k}, p_{k}\right)$ is a 
subframework of $\left(G_{k+1}, p_{k+1}\right)$ for each $k \in \mathbb{N}$. The linear maps

$$
\rho_{j, k}: \mathcal{F}\left(G_{k}, p_{k}\right) \rightarrow \mathcal{F}\left(G_{j}, p_{j}\right)
$$

defined for all $j \leq k$ by the restriction of flexes determine an inverse system $\left(\mathcal{F}\left(G_{k}, p_{k}\right), \rho_{j, k}\right)$ with associated vector space inverse $\operatorname{limit} \underset{\longleftarrow}{\lim } \mathcal{F}\left(G_{k}, p_{k}\right)$.

Definition 3.8 A tower of bar-joint frameworks $\left\{\left(G_{k}, p_{k}\right): k \in \mathbb{N}\right\}$ has the flex cancellation property if for each $k \in \mathbb{N}$ and any non-trivial infinitesimal flex $u_{k}$ of $\left(G_{k}, p_{k}\right)$ there is an $m>k$ such that $u_{k}$ does not extend to an infinitesimal flex of $\left(G_{m}, p_{m}\right)$.

If a bar-joint framework $\left(G_{m}, p_{m}\right)$ in a tower $\left\{\left(G_{k}, p_{k}\right): k \in \mathbb{N}\right\}$ has a non-trivial infinitesimal flex $u_{m}: V\left(G_{m}\right) \rightarrow X$ which can be extended to every containing framework in the tower then we call $u_{m}$ an enduring infinitesimal flex for the tower.

Lemma 3.9 Let $\left\{\left(G_{k}, p_{k}\right): k \in \mathbb{N}\right\}$ be a tower of bar-joint frameworks in a finite dimensional normed space $(X,\|\cdot\|)$ and let $u_{1}$ be an infinitesimal flex of $\left(G_{1}, p_{1}\right)$ which is an enduring flex for the tower. Then there exists a sequence $\left\{u_{k}\right\}_{k=1}^{\infty}$ such that, for each $k \in \mathbb{N}, u_{k}$ is an infinitesimal flex of $\left(G_{k}, p_{k}\right)$ and $u_{k+1}$ is an extension of $u_{k}$.

Proof Denote by $\mathcal{F}^{(k)} \subset \mathcal{F}\left(G_{k}, p_{k}\right)$ the vector space of all infinitesimal flexes $u \in$ $\mathcal{F}\left(G_{k}, p_{k}\right)$ with the property that there exists a scalar $\lambda \in \mathbb{K}$ such that $u(v)=\lambda u_{1}(v)$ for all $v \in V\left(G_{1}\right)$. Let $\rho_{k}: \mathcal{F}^{(k)} \rightarrow \mathcal{F}^{(2)}$ be the restriction map and note that since $u_{1}$ is an enduring flex we have a decreasing chain of non-zero finite dimensional linear spaces

$$
\mathcal{F}^{(2)} \supseteq \rho_{3}\left(\mathcal{F}^{(3)}\right) \supseteq \rho_{4}\left(\mathcal{F}^{(4)}\right) \supseteq \rho_{5}\left(\mathcal{F}^{(5)}\right) \supseteq \cdots
$$

Thus there exists $m \in \mathbb{N}$ such that $\rho_{n}\left(\mathcal{F}^{(n)}\right)=\rho_{m}\left(\mathcal{F}^{(m)}\right)$ for all $n>m$. Since $u_{1}$ is non-trivial and enduring there is a necessarily non-trivial extension $\tilde{u}_{m}$ say in $\mathcal{F}^{(m)}$. Let $u_{2}$ be the restriction of $\tilde{u}_{m}$ to $\left(G_{2}, p_{2}\right)$. Note that $u_{2}$ is an enduring flex since for each $n>m$ we have $u_{2} \in \rho_{m}\left(\mathcal{F}^{(m)}\right)=\rho_{n}\left(\mathcal{F}^{(n)}\right)$. Also $u_{2}$ is an extension of $u_{1}$. An induction argument can now be applied to obtain a sequence of consecutive extensions $u_{k} \in \mathcal{F}\left(G_{k}, p_{k}\right)$.

A bar-joint framework $(G, p)$ contains a tower $\left\{\left(G_{k}, p_{k}\right): k \in \mathbb{N}\right\}$ if $\left(G_{k}, p_{k}\right)$ is a subframework of $(G, p)$ for each $k \in \mathbb{N}$. A tower in $(G, p)$ is vertex-complete if $V(G)=\bigcup_{k \in \mathbb{N}} V\left(G_{k}\right)$ and edge-complete if $E(G)=\bigcup_{k \in \mathbb{N}} E\left(G_{k}\right)$. If a tower is edge-complete then the vector space $\mathcal{F}(G, p)$ of infinitesimal flexes is naturally isomorphic to the vector space inverse limit,

$$
\mathcal{F}(G, p) \cong \lim _{\longleftarrow} \mathcal{F}\left(G_{k}, p_{k}\right)
$$

Proposition 3.10 Let $(G, p)$ be a countable bar-joint framework in a finite dimensional normed space $(X,\|\cdot\|)$. If $(G, p)$ is infinitesimally rigid then every edgecomplete tower in $(G, p)$ has the flex cancellation property. 
Proof Suppose there exists an edge-complete tower $\left\{\left(G_{k}, p_{k}\right): k \in \mathbb{N}\right\}$ of finite frameworks in $(G, p)$ which does not have the flex cancellation property. Then there exists a non-trivial infinitesimal flex of some $\left(G_{k}, p_{k}\right)$ which is an enduring flex for the tower. We may assume without loss of generality that $k=1$. By Lemma 3.9 there is a sequence of infinitesimal flexes $u_{1}, u_{2}, u_{3}, \ldots$ for the chain with each flex extending the preceding flex. The tower is edge-complete and so this sequence defines an infinitesimal flex $u$ for $(G, p)$ by setting $u(v)=u_{k}(v)$ for all $v \in V\left(G_{k}\right)$ and all $k \in \mathbb{N}$. Since $u_{1}$ is a non-trivial infinitesimal flex of $\left(G_{1}, p_{1}\right)$ the flex $u$ is a non-trivial infinitesimal flex of $(G, p)$.

Remark 3.11 The key Lemma 3.9 is reminiscent of the compactness principle for locally finite structures to the effect that certain properties prevailing for all finite substructures hold also for the infinite structure. For example the $k$-colourability of a graph is one such property. See Nash-Williams [27].

We can now establish the connection between relative rigidity, flex cancellation and infinitesimal rigidity for countable bar-joint frameworks.

Definition 3.12 A tower of bar-joint frameworks $\left\{\left(G_{k}, p_{k}\right): k \in \mathbb{N}\right\}$ is relatively infinitesimally rigid if $\left(G_{k}, p_{k}\right)$ is relatively infinitesimally rigid in $\left(G_{k+1}, p_{k+1}\right)$ for each $k \in \mathbb{N}$.

Lemma 3.13 Let $\left\{\left(G_{k}, p_{k}\right): k \in \mathbb{N}\right\}$ be a framework tower in a finite dimensional normed space $(X,\|\cdot\|)$. If $\left\{\left(G_{k}, p_{k}\right): k \in \mathbb{N}\right\}$ has the flex cancellation property then there exists an increasing sequence $\left(m_{k}\right)_{k=1}^{\infty}$ of natural numbers such that the tower $\left\{\left(G_{m_{k}}, p_{m_{k}}\right): k \in \mathbb{N}\right\}$ is relatively infinitesimally rigid.

Proof Let $\mathcal{F}^{(k)} \subset \mathcal{F}\left(G_{1}, p_{1}\right)$ denote the set of all infinitesimal flexes of $\left(G_{1}, p_{1}\right)$ which extend to $\left(G_{k}, p_{k}\right)$ but not $\left(G_{k+1}, p_{k+1}\right)$. Suppose there exists an increasing sequence $\left(n_{k}\right)_{k=1}^{\infty}$ of natural numbers such that $\mathcal{F}^{\left(n_{k}\right)} \neq \emptyset$ for all $k \in \mathbb{N}$. Choose an element $u_{k} \in \mathcal{F}^{\left(n_{k}\right)}$ for each $k \in \mathbb{N}$ and note that $\left\{u_{k}: k \in \mathbb{N}\right\}$ is a linearly independent set in $\mathcal{F}\left(G_{1}, p_{1}\right)$. Since $\mathcal{F}\left(G_{1}, p_{1}\right)$ is finite dimensional we have a contradiction. Thus there exists $m_{1} \in \mathbb{N}$ such that $\mathcal{F}^{(k)}=\emptyset$ for all $k \geq m_{1}$ and so $\left(G_{1}, p_{1}\right)$ is relatively infinitesimally rigid in $\left(G_{m_{1}}, p_{m_{1}}\right)$. The result now follows by an induction argument.

Theorem 3.14 Let $(G, p)$ be a countable bar-joint framework in a finite dimensional real normed linear space $(X,\|\cdot\|)$. Then the following statements are equivalent.

(i) $(G, p)$ is infinitesimally rigid.

(ii) $(G, p)$ contains a vertex-complete tower which has the flex cancellation property.

(iii) $(G, p)$ contains a vertex-complete tower which is relatively infinitesimally rigid.

Proof The implication (i) $\Rightarrow$ (ii) is a consequence of Proposition 3.10. To prove (ii) $\Rightarrow$ (iii) apply Lemma 3.13. We now prove (iii) $\Rightarrow$ (i). Let $\left\{\left(G_{k}, p_{k}\right): k \in \mathbb{N}\right\}$ be a vertex-complete tower in $(G, p)$ which is relatively infinitesimally rigid and suppose $u$ is a non-trivial infinitesimal flex of $(G, p)$. We will construct inductively a sequence $\left(\gamma_{n}\right)_{n=1}^{\infty}$ of infinitesimal rigid motions of $X$ and an increasing sequence $\left(k_{n}\right)_{n=1}^{\infty}$ of natural numbers satisfying 
1. $u(v)=\gamma_{n}(p(v))$ for all $v \in V\left(G_{k_{n}}\right)$, and,

2. $u\left(v_{k_{n+1}}\right) \neq \gamma_{n}\left(p\left(v_{k_{n+1}}\right)\right)$ for some $v_{k_{n+1}} \in V\left(G_{k_{n+1}}\right)$.

Since the tower $\left\{\left(G_{k}, p_{k}\right): k \in \mathbb{N}\right\}$ is relatively infinitesimally rigid the restriction of $u$ to $\left(G_{k}, p_{k}\right)$ is trivial for each $k \in \mathbb{N}$. Thus there exists $\gamma_{1} \in \mathcal{T}(X)$ such that $u(v)=\gamma_{1}(p(v))$ for all $v \in V\left(G_{1}\right)$. Let $k_{1}=1$. Since $u$ is non-trivial and the tower is vertex-complete there exists $k_{2}>k_{1}$ such that $u\left(v_{k_{2}}\right) \neq \gamma_{1}\left(p\left(v_{k_{2}}\right)\right.$ ) for some $v_{k_{2}} \in V\left(G_{k_{2}}\right)$. Now the restriction of $u$ to $\left(G_{k_{2}}, p_{k_{2}}\right)$ is trivial and so there exists $\gamma_{2} \in \mathcal{T}(X)$ such that $u(v)=\gamma_{2}(p(v))$ for all $v \in V\left(G_{k_{2}}\right)$. In general, given $\gamma_{n} \in \mathcal{T}(X)$ and $k_{n} \in \mathbb{N}$ we construct $\gamma_{n+1}$ and $k_{n+1}$ using the same argument.

Let $s_{n}=\gamma_{n+1}-\gamma_{n} \in \mathcal{T}(X)$. Then $s_{n}(p(v))=0$ for all $v \in V\left(G_{k_{n}}\right)$ and $s_{n}\left(p\left(v_{k_{n+1}}\right)\right) \neq 0$ for some $v_{k_{n+1}} \in V\left(G_{k_{n+1}}\right)$. Thus $\left\{s_{n}: n \in \mathbb{N}\right\}$ is a linearly independent set in $\mathcal{T}(X)$ and since $\mathcal{T}(X)$ is finite dimensional we have a contradiction. We conclude that $(G, p)$ is infinitesimally rigid.

Theorem 3.14 gives useful criteria for the determination of infinitesimal rigidity of a countable framework $(G, p)$.

Definition 3.15 A countable bar-joint framework $(G, p)$ is sequentially infinitesimally rigid if there exists a vertex-complete tower of bar-joint frameworks $\left\{\left(G_{k}, p_{k}\right)\right.$ : $k \in \mathbb{N}\}$ in $(G, p)$ such that $\left(G_{k}, p_{k}\right)$ is infinitesimally rigid for each $k \in \mathbb{N}$.

Corollary 3.16 Let $(G, p)$ be a countable bar-joint framework in a finite dimensional normed space $(X,\|\cdot\|)$. If $(G, p)$ is sequentially infinitesimally rigid then $(G, p)$ is infinitesimally rigid.

Proof If there exists a vertex-complete tower $\left\{\left(G_{k}, p_{k}\right): k \in \mathbb{N}\right\}$ in $(G, p)$ such that $\left(G_{k}, p_{k}\right)$ is infinitesimally rigid for each $k \in \mathbb{N}$ then this framework tower is relatively infinitesimally rigid. The result now follows from Theorem 3.14.

Remark 3.17 The set of placements of a countable graph with prescribed edge lengths need not be an algebraic variety even when it can be realised as a finitely parametrised set. In fact there are infinite Kempe linkages which can draw everywhere non-differentiable curves [31,35]. It follows that the Asimow-Roth proof [3] that infinitesimal rigidity implies continuous rigidity is not available for infinite graphs, and indeed this implication does not hold in this generality. A direct way to see this is given in Kastis and Power [17] through the construction of continuously flexible crystallographic bar-joint frameworks which are infinitesimally rigid by virtue of unavoidable infinite derivatives (velocities at joints) in any continuous motion.

\subsection{Generic Placements for Countable Graphs}

Let $G$ be a countably infinite simple graph and let $q \in(1, \infty)$.

Definition 3.18 A placement $p: V(G) \rightarrow \mathbb{R}^{d}$ is locally generic in $\left(\mathbb{R}^{d},\|\cdot\|_{q}\right)$ if every finite subframework of $(G, p)$ is generic.

A tower of graphs is a sequence of finite graphs $\left\{G_{k}: k \in \mathbb{N}\right\}$ such that $G_{k}$ is a subgraph of $G_{k+1}$ for each $k \in \mathbb{N}$. A countable graph $G$ contains a vertex-complete tower $\left\{G_{k}: k \in \mathbb{N}\right\}$ if each $G_{k}$ is a subgraph of $G$ and $V(G)=\bigcup_{k \in \mathbb{N}} V\left(G_{k}\right)$. 
Proposition 3.19 Every countable simple graph $G$ has a locally generic placement in $\left(\mathbb{R}^{d},\|\cdot\|_{q}\right)$ for $q \in(1, \infty)$.

Proof Let $\left\{G_{k}: k \in \mathbb{N}\right\}$ be a vertex-complete tower in $G$ and let

$$
\pi_{j, k}: \mathbb{R}^{d\left|V\left(G_{k}\right)\right|} \rightarrow \mathbb{R}^{d\left|V\left(G_{j}\right)\right|}, \quad\left(x_{v}\right)_{v \in V\left(G_{k}\right)} \mapsto\left(x_{v}\right)_{v \in V\left(G_{j}\right)}
$$

be the natural projections whenever $G_{j} \subseteq G_{k}$. By Lemma 2.7 the set of generic placements of each $G_{k}$ is an open and dense subset of $\mathbb{R}^{d\left|V\left(G_{k}\right)\right|}$. It follows by an induction argument that for each $k \in \mathbb{N}$ there exists an open ball $B\left(p_{k}, r_{k}\right)$ in $\mathbb{R}^{d\left|V\left(G_{k}\right)\right|}$ consisting of generic placements of $G_{k}$ such that $r_{k+1}<r_{k}$ and the projection $\pi_{k, k+1}\left(p_{k+1}\right)$ is contained in the open ball $B\left(p_{k}, \frac{r_{k}}{2^{k}}\right)$. For each $j \in \mathbb{N}$ the sequence $\left\{\pi_{j, k}\left(p_{k}\right)\right\}_{k=j}^{\infty}$ is a Cauchy sequence of points in $B\left(p_{j}, \frac{r_{j}}{2}\right) \subset \mathbb{R}^{d\left|V\left(G_{j}\right)\right|}$ and hence converges to a point in $B\left(p_{j}, r_{j}\right)$. Define $p: V(G) \rightarrow \mathbb{R}^{d}$ by setting

$$
p(v)=\lim _{\substack{k \rightarrow \infty \\ k \geq j}} p_{k}(v), \quad \text { for all } v \in V\left(G_{j}\right), \quad j \in \mathbb{N} .
$$

The restriction of $p$ to $V\left(G_{j}\right)$ is a generic placement of $G_{j}$ for all $j \in \mathbb{N}$ and so $p$ is a locally generic placement of $G$.

We now show that infinitesimal rigidity and sequential infinitesimal rigidity are generic properties for countable bar-joint frameworks in $\left(\mathbb{R}^{d},\|\cdot\|_{q}\right)$ for all $q \in(1, \infty)$.

Proposition 3.20 Let $(G, p)$ be a locally generic countable bar-joint framework in $\left(\mathbb{R}^{d},\|\cdot\|_{q}\right)$ where $q \in(1, \infty)$.

(i) The infinitesimal flex dimension $\operatorname{dim}_{\mathrm{fl}}(G, p)$ is constant on the set of all locally generic placements of $G$.

(ii) If $(G, p)$ is infinitesimally rigid then every locally generic placement of $G$ is infinitesimally rigid.

(iii) If $(G, p)$ is sequentially infinitesimally rigid then every locally generic placement of $G$ is sequentially infinitesimally rigid.

Proof To show (i) choose an edge complete tower $\left\{\left(G_{k}, p\right): k \in \mathbb{N}\right\}$ in $(G, p)$. Then $\mathcal{F}_{q}(G, p)$ is isomorphic to the inverse limit of the inverse system $\left(\mathcal{F}_{q}\left(G_{k}, p\right), \rho_{j, k}\right)$ and similarly $\mathcal{T}_{q}(G, p)$ is isomorphic to the inverse limit of the inverse system $\left(\mathcal{T}_{q}\left(G_{k}, p\right), \rho_{j, k}\right)$ where $\rho_{j, k}$ are restriction maps. If $p^{\prime}$ is another locally generic placement of $G$ then $\mathcal{F}_{q}\left(G_{k}, p\right)$ is isomorphic to $\mathcal{F}_{q}\left(G_{k}, p^{\prime}\right)$ and $\mathcal{T}_{q}\left(G_{k}, p\right)$ is isomorphic to $\mathcal{T}_{q}\left(G_{k}, p^{\prime}\right)$ for each $k$. Moreover, we may choose isomorphisms which give rise to an isomorphism of the corresponding inverse limits,

$$
\begin{array}{r}
\mathcal{F}_{q}(G, p) \cong \lim \mathcal{F}_{q}\left(G_{k}, p\right) \cong \lim _{\longleftarrow} \mathcal{F}_{q}\left(G_{k}, p^{\prime}\right) \cong \mathcal{F}_{q}\left(G, p^{\prime}\right), \\
\mathcal{T}_{q}(G, p) \cong \lim _{\leftarrow} \mathcal{T}_{q}\left(G_{k}, p\right) \cong \lim _{\leftarrow} \mathcal{T}_{q}\left(G_{k}, p^{\prime}\right) \cong \mathcal{T}_{q}\left(G, p^{\prime}\right) .
\end{array}
$$

In particular the infinitesimal flex dimensions agree,

$$
\operatorname{dim}_{\mathrm{fl}}(G, p)=\operatorname{dim} \mathcal{F}_{q}(G, p) / \mathcal{T}_{q}(G, p)=\operatorname{dim} \mathcal{F}_{q}\left(G, p^{\prime}\right) / \mathcal{T}_{q}\left(G, p^{\prime}\right)=\operatorname{dim}_{\mathrm{fl}}\left(G, p^{\prime}\right)
$$




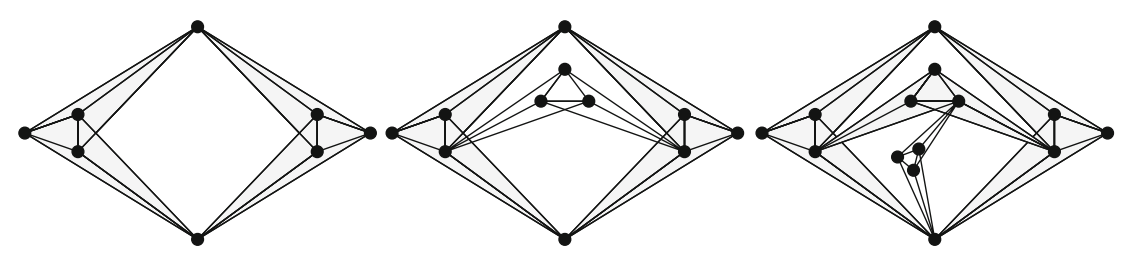

Fig. 2 The graphs $G_{1}, G_{2}$ and $G_{3}$ in Example 3.22

Statement (ii) follows immediately from (i) and (iii) holds since infinitesimal rigidity is a generic property for finite bar-joint frameworks.

The infinitesimal flex dimension of a countable graph and the classes of countable rigid and sequentially rigid graphs are now defined.

Definition 3.21 Let $G$ be a countable simple graph.

(i) $G$ is (minimally) rigid in $\left(\mathbb{R}^{d},\|\cdot\|_{q}\right)$ if the locally generic placements of $G$ are (minimally) infinitesimally rigid.

(ii) $G$ is sequentially rigid in $\left(\mathbb{R}^{d},\|\cdot\|_{q}\right)$ if the locally generic placements of $G$ are sequentially infinitesimally rigid.

(iii) The infinitesimal flexibility dimension of $G$ in $\left(\mathbb{R}^{d},\|\cdot\|_{q}\right)$ is

$$
\operatorname{dim}_{\mathrm{fl}}(G):=\operatorname{dim}_{d, q}(G):=\operatorname{dim}_{\mathrm{fl}}(G, p)=\operatorname{dim} \mathcal{F}_{q}(G, p) / \mathcal{T}_{q}(G, p) .
$$

where $p$ is any locally generic placement of $G$.

The following example demonstrates the non-equivalence of rigidity and sequential rigidity for countable graphs. The surprising fact that these properties are in fact equivalent in two dimensions is established in Theorem 4.1 below.

Example 3.22 Figure 2 illustrates the first three graphs in a tower $\left\{G_{n}: n \in \mathbb{N}\right\}$ in which $G_{n}$ is constructed inductively from a double banana graph $G_{1}$ by flex cancelling additions of copies of $K_{5} \backslash\{e\}$ (single banana graphs). The union $G$ of these graphs is a countable graph whose maximal rigid subgraphs are copies of $K_{5} \backslash\{e\}$. Thus the locally generic placements of $G$ are not sequentially infinitesimally rigid. However the tower is relatively rigid in $\left(\mathbb{R}^{3},\|\cdot\|_{2}\right)$ and so $G$ is rigid.

\section{The Equivalence of Rigidity and Sequential Rigidity}

We now prove the equivalence of rigidity and sequential rigidity for countable graphs in $\left(\mathbb{R}^{2},\|\cdot\|_{q}\right)$ for $q \in(1, \infty)$.

Theorem 4.1 Let $G$ be a countable simple graph and let $q \in(1, \infty)$. Then the following statements are equivalent.

(i) $G$ is rigid in $\left(\mathbb{R}^{2},\|\cdot\|_{q}\right)$.

(ii) $G$ is sequentially rigid in $\left(\mathbb{R}^{2},\|\cdot\|_{q}\right)$. 
Proof (i) $\Rightarrow$ (ii) Suppose $G$ is rigid in $\left(\mathbb{R}^{2},\|\cdot\|_{q}\right)$ and let $p: V(G) \rightarrow \mathbb{R}^{2}$ be a locally generic placement. By Theorem 3.14, $(G, p)$ has a vertex-complete framework tower $\left\{\left(G_{k}, p\right): k \in \mathbb{N}\right\}$ which is relatively infinitesimally rigid. By Theorem 3.6, $G_{k}$ has a rigid container $H_{k}$ in $G_{k+1}$ for each $k \in \mathbb{N}$. Thus $\left\{H_{k}: k \in \mathbb{N}\right\}$ is the required vertex-complete tower of rigid subgraphs in $G$.

(ii) $\Rightarrow$ (i) If $p: V(G) \rightarrow \mathbb{R}^{2}$ is a locally generic placement of $G$ in $\left(\mathbb{R}^{2},\|\cdot\|_{q}\right)$ then by Corollary 3.16, $(G, p)$ is infinitesimally rigid and so $G$ is rigid.

We now prove our main theorem. We use the convention that if $P$ is a property of a graph then a $P$-tower is a tower for which each graph $G_{k}$ has property $P$. Thus a $(2,3)$-tight tower is a nested sequence of subgraphs $\left\{G_{k}: k \in \mathbb{N}\right\}$ each of which is (2, 3)-tight.

Proof of Theorem 1 (i) $\Rightarrow$ (ii) If $G$ is rigid then by Theorem 4.1, $G$ is sequentially rigid and so there exists a vertex-complete tower $\left\{G_{k}: k \in \mathbb{N}\right\}$ of rigid subgraphs in $G$. We will construct a tower $\left\{H_{k}: k \in \mathbb{N}\right\}$ of $(2, l)$-tight subgraphs of $G$ satisfying $V\left(H_{k}\right)=V\left(G_{k}\right)$ for each $k \in \mathbb{N}$.

Let $H_{1}=G_{1} \backslash E_{1}$ be a minimally rigid spanning subgraph of $G_{1}$ obtained by removing a set $E_{1} \subset E\left(G_{1}\right)$ of edges from $G_{1}$. It follows on considering the rigidity matrix for a generic placement of $G_{k}$ that $G_{k} \backslash E_{1}$ is rigid for each $k \in \mathbb{N}$. Letting $G_{k}^{\prime}=G_{k} \backslash E_{1}$ for all $k \geq 2$ we obtain a vertex-complete tower of rigid subgraphs in $G$

$$
H_{1} \subset G_{2}^{\prime} \subset G_{3}^{\prime} \subset \cdots
$$

where $H_{1}$ is minimally rigid, $V\left(H_{1}\right)=V\left(G_{1}\right)$ and $V\left(G_{k}^{\prime}\right)=V\left(G_{k}\right)$ for all $k \geq 2$.

Suppose we have constructed a vertex-complete tower of rigid subgraphs in $G$,

$$
H_{1} \subset H_{2} \subset \cdots \subset H_{n} \subset G_{n+1}^{\prime} \subset G_{n+2}^{\prime} \subset \cdots,
$$

where $H_{1}, H_{2}, \ldots, H_{n}$ are minimally rigid, $V\left(H_{k}\right)=V\left(G_{k}\right)$ for each $k=1,2, \ldots, n$ and $V\left(G_{k}^{\prime}\right)=V\left(G_{k}\right)$ for all $k \geq n+1$. Let $H_{n+1}=G_{n+1} \backslash E_{n+1}$ be a minimally rigid spanning subgraph of $G_{n+1}^{\prime}$ obtained by removing a set $E_{n+1} \subset E\left(G_{n+1}^{\prime}\right)$ of edges from $G_{n+1}^{\prime}$. We can arrange that $H_{n}$ is a subgraph of $H_{n+1}$. It follows on considering the rigidity matrix for a generic placement of $G_{k}^{\prime}$ that $G_{k}^{\prime} \backslash E_{n+1}$ is rigid for each $k \geq n+1$. Replacing $G_{k}^{\prime}$ with $G_{k}^{\prime} \backslash E_{n+1}$ for each $k \geq n+2$ we obtain a vertex-complete tower in $G$,

$$
H_{1} \subset H_{2} \subset \cdots \subset H_{n+1} \subset G_{n+2}^{\prime} \subset G_{n+3}^{\prime} \subset \cdots
$$

consisting of rigid subgraphs with $H_{1}, H_{2}, \ldots, H_{n+1}$ minimally rigid, $V\left(H_{k}\right)=$ $V\left(G_{k}\right)$ for each $k=1,2, \ldots, n+1$ and $V\left(G_{k}^{\prime}\right)=V\left(G_{k}\right)$ for all $k \geq n+2$.

By induction there exists a vertex-complete tower $\left\{H_{k}: k \in \mathbb{N}\right\}$ of minimally rigid subgraphs in $G$. In case (A), Theorem 2.11 implies that each $H_{k}$ is $(2,3)$-tight and in case (B) Theorem 2.12 implies that each $H_{k}$ is $(2,2)$-tight. 
(ii) $\Rightarrow$ (i) Let $\left\{G_{k}: k \in \mathbb{N}\right\}$ be a $(2, l)$-tight vertex-complete tower in $G$. By Theorems 2.11 and 2.12, each $G_{k}$ is a rigid graph in $\left(\mathbb{R}^{2},\|\cdot\|_{q}\right)$ and so $G$ is sequentially rigid. By Theorem 4.1, $G$ is rigid.

Corollary 4.2 Let $G$ be a countable simple graph.

(A) The following statements are equivalent.

(i) $G$ is minimally rigid in $\left(\mathbb{R}^{2},\|\cdot\|_{2}\right)$.

(ii) $G$ contains a $(2,3)$-tight edge-complete tower.

(B) If $q \in(1,2) \cup(2, \infty)$ then the following statements are equivalent.

(i) $G$ is minimally rigid in $\left(\mathbb{R}^{2},\|\cdot\|_{q}\right)$.

(ii) G contains a $(2,2)$-tight edge-complete tower.

Proof (i) $\Rightarrow$ (ii) If $G$ is minimally rigid in $\left(\mathbb{R}^{2},\|\cdot\|_{q}\right)$ then by Theorem $1.1, G$ contains a $(2, l)$-tight vertex-complete tower $\left\{G_{k}: k \in \mathbb{N}\right\}$ and this tower must be edge-complete.

(ii) $\Rightarrow$ (i) If $G$ contains a $(2, l)$-tight edge-complete tower $\left\{G_{k}: k \in \mathbb{N}\right\}$ then by Theorem 1.1, G is rigid. Let $v w \in E(G)$ and suppose $G \backslash\{v w\}$ is rigid. By Theorem 4.1, $G \backslash\{v w\}$ is sequentially rigid and so there exists a vertex-complete tower $\left\{H_{k}\right.$ : $k \in \mathbb{N}\}$ in $G \backslash\{v w\}$ consisting of rigid subgraphs. Choose a sufficiently large $k$ such that $v, w \in V\left(H_{k}\right)$ and choose a sufficiently large $n$ such that $v w \in E\left(G_{n}\right)$ and $H_{k}$ is a subgraph of $G_{n}$. Then $H_{k} \cup\{v w\}$ is a subgraph of $G_{n}$ which fails the sparsity count for $G_{n}$. We conclude that $G \backslash\{v w\}$ is not rigid in $\left(\mathbb{R}^{2},\|\cdot\|_{q}\right)$ for all $v w \in E(G)$.

Note that it follows from this corollary that the graph of a minimally infinitesimally rigid framework may have some or all of its vertices of countable degree.

\subsection{Remarks and Open Problems}

One can also take a matroidal point of view for infinitesimally rigid frameworks and define the infinite matroid $\mathcal{R}_{2}^{\infty}$ (resp. $\mathcal{R}_{2, q}^{\infty}$ ) on the set $S$ of edges of the countable complete graph $K_{\infty}$. The independent sets in this matroid are the subsets of edges of a sequential Laman graph (resp. sequentially $(2,2)$-tight graph). Our results show that these matroids are finitary (see Oxley [33] and Bruhn et al. [6]) and so are closely related to their finite matroid counterparts.

It is a long-standing open problem to characterise in combinatorial terms the finite simple 3-rigid graphs despite progress in understanding the corresponding rigidity matroid $\mathcal{R}_{3}$. See Cheng and Sitharam [7] for example. However the absence of rotational isometries in the non-Euclidean spaces $\left(\mathbb{R}^{3},\|\cdot\|_{q}\right)$ suggests that a combinatorial characterisation of finite rigid graphs might be possible in terms of $(3,3)$-tight graphs. If this is so then part (B) of Theorem 1.1 would extend to $d=3$.

We note that there are a number of further directions and natural problems in which relative rigidity methods play a role.

(i) It is well-known that generic body-bar frameworks are more tractable than barjoint frameworks and in the next section we obtain variants of Tay's [40] celebrated combinatorial characterisation. 
(ii) Finite bar-joint frameworks in three dimensions whose joints are constrained to move on an algebraic surface are considered in $[28,29]$. In particular the graphs for generically minimally infinitesimally rigid frameworks for the cylinder are the $(2,2)$-tight graphs. The methods and results above for $\left(\mathbb{R}^{2},\|\cdot\|_{q}\right)$ carry over readily to the cylinder.

(iii) An important theme and proof technique in the rigidity of finite graphs and geometric systems is the use of inductive constructions, that is, the construction of all graphs in a combinatorial class through a finite number of elementary construction moves, such as Henneberg moves. In our companion paper Kitson and Power [21] we consider such constructions for infinite graphs and for infinitely faceted polytopes.

(iv) In [19] it is shown that relative infinitesimal rigidity with respect to a polyhedral norm on $\mathbb{R}^{d}$ may be determined from an edge-labelling induced by the framework placement. This provides a convenient tool which is applied to obtain an analogue of Laman's theorem for polyhedral norms on $\mathbb{R}^{2}$. The passage to countable graphs differs from the present case in that the notion of a locally generic placement used here for $\ell^{q}$ norms is no longer appropriate in the case of a polyhedral norm. Thus an analogue of Theorem 1.1 is not available, however, Theorem 3.14 may still be applied for all polyhedral norms on $\mathbb{R}^{d}$.

(v) Globally rigid graphs are those graphs $G$ whose generic frameworks $(G, p)$ admit no equivalent non-congruent realisations. There have been a number of recent significant advances in the determination of such graphs [11,14] and it would be of interest to extend such results to countable graphs.

\section{Rigidity of Multi-body Graphs}

Tay's theorem [40] provides a combinatorial characterisation of the finite multi-graphs without reflexive edges which have infinitesimally rigid generic realisations as bodybar frameworks in Euclidean space. In this section we extend Tay's characterisation to countable multi-graphs and obtain analogues of both characterisations for the nonEuclidean $\ell^{q}$ norms for all dimensions $d \geq 2$.

\subsection{Tay's Theorem and Non-Euclidean Rigidity}

We now consider bar-joint frameworks in $\left(\mathbb{R}^{d},\|\cdot\|_{q}\right)$, where $q \in(1, \infty)$, which arise from the following class of simple graphs.

Definition 5.1 A multi-body graph for $\left(\mathbb{R}^{d},\|\cdot\|_{q}\right)$ is a finite or countable simple graph $G$ for which there exists a vertex partition

$$
V(G)=\bigcup_{k} V_{k}
$$

consisting of a finite or countable collection of subsets $V_{k}$ such that for each $k$,

1. the vertex-induced subgraph determined by $V_{k}$ is a rigid graph in $\left(\mathbb{R}^{d},\|\cdot\|_{q}\right)$, and,

2. every vertex $v \in V_{k}$ is adjacent to at most one vertex in $V(G) \backslash V_{k}$. 
The rigid vertex-induced subgraph determined by $V_{k}$ is denoted $B_{k}$ and is called a body of $G$. An edge $v w \in E(G)$ which is incident with vertices from two distinct bodies $B_{i}$ and $B_{j}$ is called an inter-body edge. Thus a multi-body graph is composed of pairwise vertex-disjoint bodies together with inter-body edges such that no pair of inter-body edges of $G$ shares a vertex.

Each multi-body graph $G$ has an associated finite or countable body-bar graph $G_{b}=\left(V\left(G_{b}\right), E\left(G_{b}\right)\right)$ which is the multi-graph with vertex set labelled by the bodies of $G$ and with edge set derived from the inter-body edges of $G$.

Tay's theorem may be restated as follows:

Theorem 5.2 (Tay [40]) Let $G$ be a finite multi-body graph for $\left(\mathbb{R}^{d},\|\cdot\|_{2}\right)$ and suppose that $G$ contains at least two bodies. Then the following statements are equivalent.

(i) $G$ is rigid in Euclidean space $\left(\mathbb{R}^{d},\|\cdot\|_{2}\right)$.

(ii) $G_{b}$ contains a $\left(\frac{d(d+1)}{2}, \frac{d(d+1)}{2}\right)$-tight spanning subgraph.

The following lemma shows that the bodies $B_{1}, B_{2}, \ldots$ of a multi-body graph $G$ may be modelled in a number of different ways without altering the rigidity properties of $G$.

Lemma 5.3 Let $G$ and $G^{\prime}$ be two finite multi-body graphs for $\left(\mathbb{R}^{d},\|\cdot\|_{q}\right)$ with isomorphic body-bar graphs and $q \in(1, \infty)$. Then $\operatorname{dim}_{d, q}(G)=\operatorname{dim}_{d, q}\left(G^{\prime}\right)$.

Proof Choose a multi-body graph $H$ with body-bar graph $H_{b}$ isomorphic to $G_{b}$ and $G_{b}^{\prime}$ such that each body of $H$ is a complete graph with more vertices than the corresponding bodies of $G$ and $G^{\prime}$. Then there exist natural graph homomorphisms $\phi: G \rightarrow H$ and $\phi^{\prime}: G^{\prime} \rightarrow H$. If $p_{H}: V(H) \rightarrow \mathbb{R}^{d}$ is a generic placement of $H$ then $p: V(G) \rightarrow \mathbb{R}^{d}$ defined by $p_{v}=\left(p_{H}\right)_{\phi(v)}$ is a generic placement of $G$. Now the linear mapping $A: \mathcal{F}_{q}\left(H, p_{H}\right) \rightarrow \mathcal{F}_{q}(G, p), A(u)_{v}=u_{\phi(v)}$, is an isomorphism. Applying the same argument to $G^{\prime}$ we obtain a generic placement $p: V\left(G^{\prime}\right) \rightarrow \mathbb{R}^{d}$ and a linear isomorphism $A^{\prime}: \mathcal{F}_{q}\left(H, p_{H}\right) \rightarrow \mathcal{F}_{q}\left(G^{\prime}, p^{\prime}\right)$. The result follows.

Example 5.4 The complete graph $K_{d+1}$ is $\left(d, \frac{d(d+1)}{2}\right)$-tight and is minimally rigid for $\left(\mathbb{R}^{d},\|\cdot\|_{2}\right)$. The complete graph $K_{2 d}$ is $(d, d)$-tight and is a minimally rigid graph for $\left(\mathbb{R}^{d},\|\cdot\|_{q}\right)$ for each of the non-Euclidean $\ell^{q}$-norms. These sparsity and rigidity properties persist for graphs obtained from these complete graphs by a finite sequence of Henneberg vertex extension moves of degree $d$. Thus we may assume without loss of generality that the bodies of a finite multi-body graph for $\left(\mathbb{R}^{d},\|\cdot\|_{q}\right)$ are $\left(d, \frac{d(d+1)}{2}\right)$-tight in the Euclidean case and $(d, d)$-tight in the non-Euclidean case. The convenience of modelling multi-body graphs in this way is that the combinatorial and $\ell^{q}$-norm analysis of earlier sections is ready-to-hand.

There is a natural vertex-induced surjective graph homomorphism $\pi: G \rightarrow \bar{G}_{b}$ where $\bar{G}_{b}$ is the multi-graph obtained by contracting the bodies of $G$. The body-bar graph $G_{b}$ is a subgraph of $\bar{G}_{b}$ obtained by removing reflexive edges and $\pi$ gives a bijection between the inter-body edges of $G$ and the edges of $G_{b}$.

Theorem 5.5 Let $G$ be a finite multi-body graph for $\left(\mathbb{R}^{d},\|\cdot\|_{q}\right)$ where $q \in(1,2) \cup$ $(2, \infty)$. Then the following statements are equivalent. 
(i) $G$ is rigid in $\left(\mathbb{R}^{d},\|\cdot\|_{q}\right)$.

(ii) $G_{b}$ has a $(d, d)$-tight spanning subgraph.

Proof (i) $\Rightarrow$ (ii) We can assume without loss of generality that each body of $G$ is $(d, d)$ tight. Suppose that $G$ is minimally rigid in $\left(\mathbb{R}^{d},\|\cdot\|_{q}\right)$ with bodies $B_{1}, B_{2}, \ldots, B_{n}$. If $G_{b}$ is the body-bar graph for $G$ then $\left|V\left(G_{b}\right)\right|=n$ and we have

$$
\begin{aligned}
\left|E\left(G_{b}\right)\right| & =|E(G)|-\sum_{i=1}^{n}\left|E\left(B_{i}\right)\right| \\
& =(d|V(G)|-d)-\sum_{i=1}^{n}\left(d\left|V\left(B_{i}\right)\right|-d\right)=d\left|V\left(G_{b}\right)\right|-d .
\end{aligned}
$$

Let $H_{b}$ be a subgraph of $G_{b}$ and let $\pi: G \rightarrow \bar{G}_{b}$ be the natural graph homomorphism. Define $H$ to be the subgraph of $G$ with $V(H)=\pi^{-1}\left(V\left(H_{b}\right)\right)$ such that $H$ contains the body $B_{i}$ whenever $\pi\left(V\left(B_{i}\right)\right) \in V\left(H_{b}\right)$ and $H$ contains the inter-body edge $v w$ whenever $\pi(v) \pi(w) \in E\left(H_{b}\right)$. Then $\left|V\left(H_{b}\right)\right|=|\mathcal{I}|$ where $\mathcal{I}=\{i \in\{1, \ldots, n\}$ : $\left.B_{i} \subset H\right\}$ and

$$
\begin{aligned}
\left|E\left(H_{b}\right)\right| & =|E(H)|-\sum_{i \in \mathcal{I}}\left|E\left(B_{i}\right)\right| \\
& \leq(d|V(H)|-d)-\sum_{i \in \mathcal{I}}\left(d\left|V\left(B_{i}\right)\right|-d\right)=d\left|V\left(H_{b}\right)\right|-d .
\end{aligned}
$$

Thus $G_{b}$ is $(d, d)$-tight. For the general case note that by removing edges from $G$ we obtain a minimally rigid multi-body graph $\tilde{G}$. Thus by the above argument $\tilde{G}_{b}$ is a vertex-complete $(d, d)$-tight subgraph of $G_{b}$.

(ii) $\Rightarrow$ (i) If $G_{b}$ is $(d, d)$-tight then it admits a partition as an edge-disjoint union of $d$ spanning trees $T_{1}, T_{2}, \ldots, T_{d}$ (see [26]). We will construct a placement of $G$ so that $p_{v}-p_{w}$ lies on the $i$ th coordinate axis in $\mathbb{R}^{d}$ whenever $v w$ is an inter-body edge with $\pi(v w) \in T_{i}$.

By Lemma 5.3 we can assume that the bodies $B_{1}, B_{2}, \ldots, B_{n}$ of $G$ are copies of the complete graph $K_{m}$ for some sufficiently large $m$. Let $p_{1}: V\left(B_{1}\right) \rightarrow \mathbb{R}^{d}$ be a generic placement of the body $B_{1}$ and define inductively the placements $p_{k}: V\left(B_{k}\right) \rightarrow \mathbb{R}^{d}$ for $k=2, \ldots, n$ so that

(1) $p_{k}\left(V\left(B_{k}\right)\right)=p_{1}\left(V\left(B_{1}\right)\right)$, and,

(2) $p_{j}(v)=p_{k}(w)$ whenever $j<k$ and $v w \in E(G)$ is an inter-body edge with $v \in V\left(B_{j}\right)$ and $w \in V\left(B_{k}\right)$.

Then $\left(B_{k}, p_{k}\right)$ is a generic, and hence infinitesimally rigid, bar-joint framework for each $k=1,2, \ldots, n$.

Define $p: V(G) \rightarrow \mathbb{R}^{d}$ by setting $p(v)=p_{i}(v)$ whenever $v \in V\left(B_{i}\right)$. Note that $p$ is not a placement of $G$ since $p_{v}=p_{w}$ for each inter-body edge $v w \in E(G)$. However, by perturbing $p$ by a small amount we can obtain a placement $p^{\prime}$. Let $\epsilon>0$ and let $e_{1}, e_{2}, \ldots, e_{d}$ be the usual basis in $\mathbb{R}^{d}$. If $v \in V(G)$ is not incident with an inter-body 
edge then set $p_{v}^{\prime}=p_{v}$. If $v w \in E(G)$ is an inter-body edge and $\pi(v w) \in T_{i}$ then let $p_{v}^{\prime}=p_{v}+\epsilon e_{i}$ and $p_{w}^{\prime}=p_{w}$. The rigidity matrix for $\left(G, p^{\prime}\right)$ has the form

$$
R_{q}\left(G, p^{\prime}\right)=\left[\begin{array}{lll}
R_{q}\left(B_{1}, p^{\prime}\right) & & \\
& \ddots & \\
& & R_{q}\left(B_{n}, p^{\prime}\right) \\
\hline & Z
\end{array}\right],
$$

where the rows of the submatrix $Z$ correspond to the inter-body edges in $G$.

Suppose $u=\left(u_{1}, \ldots, u_{n}\right) \in \operatorname{ker} R_{q}\left(G, p^{\prime}\right)$. For a sufficiently small $\epsilon$ each subframework $\left(B_{i}, p^{\prime}\right)$ is infinitesimally rigid, and so $u_{i}=\left(a_{i}, \ldots, a_{i}\right)$ for some $a_{i} \in \mathbb{R}^{d}$. If $v w$ is an inter-body edge with $\pi(v w) \in T_{i}$ then the corresponding row entries in $R_{q}\left(G, p^{\prime}\right)$ are non-zero in the $p_{v, i}$ and $p_{w, i}$ columns only. The spanning tree property now ensures that $a_{1}=\cdots=a_{n}$ and so the kernel of $R_{q}\left(G, p^{\prime}\right)$ has dimension $d$. Thus $p^{\prime}$ is an infinitesimally rigid placement of $G$ in $\left(\mathbb{R}^{d},\|\cdot\|_{q}\right)$. More generally if $G_{b}$ contains a vertex-complete $(d, d)$-tight subgraph then by the above argument $G$ is rigid in $\left(\mathbb{R}^{d},\|\cdot\|_{q}\right)$.

A key feature of body-bar frameworks is the non-incidence condition for the bars. This makes available special realisations which are rigid, as we have seen in the proof of the analogue of Tay's theorem, Theorem 5.5. Other instances of this can be seen in the matroid analysis of Whiteley [44] and in the analysis of Borcea and Streinu [5] and Ross [36] of locally finite graphs with periodically rigid periodic bar-joint frameworks.

We will require the following definition and corollary to characterise the countable rigid multi-body graphs for $\left(\mathbb{R}^{d},\|\cdot\|_{q}\right)$.

Definition 5.6 A multi-body graph for $\left(\mathbb{R}^{d},\|\cdot\|_{q}\right)$ is essentially minimally rigid if it is rigid and removing any inter-body edge results in a multi-body graph which is not rigid.

Corollary 5.7 Let $G$ be a finite multi-body graph for $\left(\mathbb{R}^{d},\|\cdot\|_{q}\right)$ and suppose that one of the following conditions holds.

(a) $q=2$ and $k=\frac{d(d+1)}{2}$, or,

(b) $q \in(1,2) \cup(2, \infty)$ and $k=d$.

Then the following statements are equivalent.

(i) $G$ is essentially minimally rigid in $\left(\mathbb{R}^{d},\|\cdot\|_{q}\right)$.

(ii) $G_{b}$ is a $(k, k)$-tight multi-graph.

Proof The proof follows immediately from Theorem 5.2 in case (a) and from Theorem 5.5 in case (b).

\subsection{Rigidity of Countable Multi-body Graphs}

We are now able to characterise the countable rigid multi-body graphs in $\left(\mathbb{R}^{d},\|\cdot\|_{q}\right)$ for all dimensions $d \geq 2$ and all $q \in(1, \infty)$. Given a finite bar-joint framework $(G, p)$ in $\left(\mathbb{R}^{d},\|\cdot\|_{q}\right)$ we denote by $X_{\text {row }}(G, p)$ the row space of the rigidity matrix $R_{q}(G, p)$. 
Definition 5.8 A finite multi-body graph for $\left(\mathbb{R}^{d},\|\cdot\|_{q}\right)$ is essentially independent if given any generic placement $p \in \mathcal{P}(G)$ the row space of the rigidity matrix $R_{q}(G, p)$ may be expressed as a direct sum

$$
X_{\text {row }}(G, p)=X_{B_{1}} \oplus \cdots \oplus X_{B_{n}} \oplus X_{\mathrm{IB}}
$$

where $X_{B_{i}}$ is the subspace of $X_{\text {row }}(G, p)$ spanned by the rows of $R_{q}(G, p)$ which correspond to the edges of the body $B_{i}$ and $X_{\mathrm{IB}}$ is the subspace spanned by the rows which correspond to the inter-body edges of $G$.

The following result is an analogue of Proposition 3.5.

Proposition 5.9 Let $G$ be a finite multi-body graph for $\left(\mathbb{R}^{d},\|\cdot\|_{q}\right)$ and suppose that one of the following conditions holds.

(a) $q=2, k=\frac{d(d+1)}{2}$ and $G$ contains at least $d(d+1)$ vertices, or,

(b) $q \in(1,2) \cup(2, \infty), k=d$ and $G$ contains at least $2 d$ vertices.

Then the following statements are equivalent.

(i) $G$ is essentially independent with respect to $\left(\mathbb{R}^{d},\|\cdot\|_{q}\right)$.

(ii) $G_{b}$ is $(k, k)$-sparse.

Proof Suppose $G$ is essentially independent and let $p: V(G) \rightarrow \mathbb{R}^{d}$ be a generic placement of $G$. If $H_{b}$ is a subgraph of $G_{b}$ and $B_{1}, B_{2}, \ldots, B_{n}$ are the bodies of $G$ then let $H$ be the subgraph of $G$ with $V(H)=\pi^{-1}\left(V\left(H_{b}\right)\right)$ such that $H$ contains the body $B_{i}$ whenever $\pi\left(V\left(B_{i}\right)\right) \in V\left(H_{b}\right)$ and $H$ contains the inter-body edge $v w$ whenever $\pi(v w) \in E\left(H_{b}\right)$. If $\mathcal{I}=\left\{i \in\{1, \ldots, n\}: B_{i} \subset H\right\}$ then

$$
\begin{aligned}
\left|E\left(H_{b}\right)\right| & =\operatorname{rank} R_{q}(H, p)-\sum_{i \in \mathcal{I}} \operatorname{rank} R_{q}\left(B_{i}, p\right) \\
& \leq(d|V(H)|-k)-\sum_{i \in \mathcal{I}}\left(d\left|V\left(B_{i}\right)\right|-k\right)=k\left|V\left(H_{b}\right)\right|-k
\end{aligned}
$$

Thus $G_{b}$ is $(k, k)$-sparse.

Conversely, if $G_{b}$ is $(k, k)$-sparse then by Lemma 3.2, $G_{b}$ is a vertex-complete subgraph of a $(k, k)$-tight multi-graph $G_{b}^{\prime}$ which has no reflexive edges. Let $G^{\prime}$ be a multi-body graph with body-bar graph isomorphic to $G_{b}^{\prime}$ and which contains $G$ as a subgraph. By Corollary 5.7, $G^{\prime}$ is essentially minimally rigid and it follows that $G$ is essentially independent.

We now prove an analogue of Theorem 3.6 which shows that in the category of multi-body graphs relative rigidity is equivalent to the existence of a rigid container for all dimensions $d$ and for all $\ell^{q}$ norms.

Theorem 5.10 Let $G$ be a finite multi-body graph for $\left(\mathbb{R}^{d},\|\cdot\|_{q}\right)$ and let $H$ be a subgraph of $G$ which is a multi-body graph whose body subgraphs are bodies of $G$. Suppose that one of the following conditions holds. 
(a) $q=2$ and $H$ contains at least $d(d+1)$ vertices, or,

(b) $q \in(1,2) \cup(2, \infty)$ and $H$ contains at least $2 d$ vertices.

Then the following statements are equivalent.

(i) $H$ is relatively rigid in $G$ with respect to $\left(\mathbb{R}^{d},\|\cdot\|_{q}\right)$.

(ii) $H$ has a rigid container in $G$ with respect to $\left(\mathbb{R}^{d},\|\cdot\|_{q}\right)$ which is a multi-body graph.

Proof (i) $\Rightarrow$ (ii) Consider first the case when $G$ is essentially independent with respect to $\left(\mathbb{R}^{d},\|\cdot\|_{q}\right)$. By Proposition 5.9 , the body-bar graph $G_{b}$ is $(k, k)$-sparse, for the appropriate value of $k$. Let $\pi(v), \pi(w) \in V\left(H_{b}\right)$ be distinct vertices of $H_{b}$ with $\pi(v w) \notin E\left(H_{b}\right)$. By enlarging the bodies of $G$ and $H$ we can assume without loss of generality that there exist representative vertices $v, w \in V(H)$ such that $v$ and $w$ are not incident with any inter-body edges of $G$. Since $K_{V(H)}$ is rigid in $\left(\mathbb{R}^{d},\|\cdot\|_{q}\right)$ the relative rigidity property implies that

$$
\mathcal{F}_{q}(G, p)=\mathcal{F}_{q}\left(G \cup K_{V(H)}, p\right)
$$

for every generic placement $p$. It follows that $G^{\prime}=G \cup\{v w\}$ is a multi-body graph which is not essentially independent. Note that $G^{\prime}$ has the same bodies as $G$ and so by Proposition 5.9, the associated body-bar graph $\left(G^{\prime}\right)_{b}=G_{b} \cup\{\pi(v w)\}$ is not $(k, k)$ sparse where $\pi: G \rightarrow \bar{G}_{b}$ is the natural graph homomorphism. Thus by Lemma 3.1 there exists a $(k, k)$-tight subgraph $\left(H_{v, w}\right)_{b}$ of $G_{b}$ with $\pi(v), \pi(w) \in V\left(\left(H_{v, w}\right)_{b}\right)$. Let $H_{v, w}$ be the induced multi-body subgraph of $G$ with body-bar graph isomorphic to $\left(H_{v, w}\right)_{b}$. By Corollary $5.7, H_{v, w}$ is rigid in $\left(\mathbb{R}^{2},\|\cdot\|_{q}\right)$.

Define $H^{\prime}$ to be the union of $H$ together with the subgraphs $H_{v, w}$ for all such pairs $\pi(v), \pi(w) \in V\left(H_{b}\right)$. Thus $H^{\prime}$ is the multi-body subgraph of $G$ with body-bar graph isomorphic to $H_{b}^{\prime}$. Then $H^{\prime}$ is rigid in $\left(\mathbb{R}^{d},\|\cdot\|_{q}\right)$ and so $H^{\prime}$ is a rigid container for $H$ in $G$.

If $G$ is not essentially independent then let $p: V(G) \rightarrow \mathbb{R}^{d}$ be a generic placement of $G$. There exists an inter-body edge $v w \in E(G)$ such that

$$
\operatorname{ker} R_{q}(G, p)=\operatorname{ker} R_{q}(G \backslash\{v w\}, p) \text {. }
$$

Let $G_{1}=G \backslash\{v w\}$ and $H_{1}=H \backslash\{v w\}$ and note that $H_{1}$ is relatively rigid in $G_{1}$. Continuing to remove edges in this way we arrive after finitely many iterations at subgraphs $H_{n}$ and $G_{n}$ such that $V\left(H_{n}\right)=V(H), H_{n}$ is relatively rigid in $G_{n}$ and $G_{n}$ is essentially independent. By the above argument there exists a rigid container $H_{n}^{\prime}$ for $H_{n}$ in $G_{n}$. Now $H^{\prime}=H_{n}^{\prime} \cup H$ is a rigid container for $H$ in $G$.

(ii) $\Rightarrow$ (i) If $H$ has a rigid container $H^{\prime}$ in $G$ and $p: V(G) \rightarrow \mathbb{R}^{d}$ is a generic placement of $G$ then no non-trivial infinitesimal flex of $(H, p)$ extends to $\left(H^{\prime}, p\right)$. The result follows.

We now prove the equivalence of rigidity and sequential rigidity for multi-body graphs with respect to all $\ell^{q}$-norms and in all dimensions $d \geq 2$.

Theorem 5.11 Let $G$ be a countable multi-body graph for $\left(\mathbb{R}^{d},\|\cdot\|_{q}\right)$ where $q \in$ $(1, \infty)$. The following statements are equivalent. 
(i) $G$ is rigid in $\left(\mathbb{R}^{d},\|\cdot\|_{q}\right)$.

(ii) $G$ is sequentially rigid in $\left(\mathbb{R}^{d},\|\cdot\|_{q}\right)$.

Proof Suppose $G$ is rigid in $\left(\mathbb{R}^{d},\|\cdot\|_{q}\right)$ and let $p: V(G) \rightarrow \mathbb{R}^{d}$ be a locally generic placement. By Theorem 3.14, there exists a vertex-complete tower $\left\{\left(G_{k}, p_{k}\right): k \in \mathbb{N}\right\}$ in $(G, p)$ which is relatively infinitesimally rigid. Moreover, we can assume that each $G_{k}$ is a multi-body graph. By Proposition 5.10, $G_{k}$ has a rigid container $H_{k}$ in $G_{k+1}$ for each $k \in \mathbb{N}$. Thus the sequence $\left\{H_{k}: k \in \mathbb{N}\right\}$ is a vertex-complete tower of rigid graphs in $G$. For the converse apply Corollary 3.16 .

We now prove our second main result which generalises Tay's theorem to countable multi-body graphs.

Proof of Theorem 2 (i) $\Rightarrow$ (ii) If $G$ is rigid then by Theorem 5.11, $G$ is sequentially rigid. Let $\left\{G_{k}: k \in \mathbb{N}\right\}$ be a vertex-complete tower of rigid subgraphs in $G$ and let $B_{1}, B_{2}, \ldots$ be the bodies of $G$. We may assume that each $G_{k}$ is a multi-body graph. Applying the induction argument used in Theorem 1.1 we construct a vertex-complete tower of essentially minimally rigid multi-body subgraphs in $G$. To do this let $H_{1}$ be the multi-body graph obtained by taking all bodies which lie in $\tilde{G}_{1}$ and adjoining interbody edges of $G_{1}$ until an essentially minimally rigid graph is reached. The induced sequence of body-bar graphs $\left\{\left(H_{k}\right)_{b}: k \in \mathbb{N}\right\}$ is a vertex-complete tower in $G_{b}$. By Corollary 5.7 each body-bar graph $\left(H_{k}\right)_{b}$ is $\left(\frac{d(d+1)}{2}, \frac{d(d+1)}{2}\right)$-tight in case (A) and $(d, d)$-tight in case $(\mathrm{B})$.

(ii) $\Rightarrow$ (i) Let $\left\{G_{k, b}: k \in \mathbb{N}\right\}$ be a $\left(\frac{d(d+1)}{2}, \frac{d(d+1)}{2}\right)$-tight vertex-complete tower in $G_{b}$ and let $\pi: G \rightarrow \bar{G}_{b}$ be the natural graph homomorphism. Define $G_{k}$ to be the subgraph of $G$ with $V\left(G_{k}\right)=\pi^{-1}\left(V\left(G_{k, b}\right)\right)$ such that $G_{k}$ contains the body $B_{i}$ whenever $\pi\left(V\left(B_{i}\right)\right) \in V\left(G_{k, b}\right)$ and $G_{k}$ contains the inter-body edge $v w$ whenever $\pi(v w) \in E\left(G_{k, b}\right)$. Then $G_{k, b}$ is the body-bar graph for $G_{k}$ and so $G_{k}$ is rigid by Theorem 5.2. Thus $\left\{G_{k}: k \in \mathbb{N}\right\}$ is a vertex-complete tower of rigid subgraphs in $G$ and so $G$ is sequentially rigid. By Theorem 5.11, $G$ is rigid.

To prove (B) we apply similar arguments to the above using the non-Euclidean versions of the relevant propositions and substituting Theorem 5.5 for Theorem 5.2.

Corollary 5.12 Let $G$ be a countable multi-body graph for $\left(\mathbb{R}^{d},\|\cdot\|_{q}\right)$ where $q \in$ $(1, \infty)$.

(A) The following statements are equivalent.

(i) $G$ is essentially minimally rigid in $\left(\mathbb{R}^{d},\|\cdot\|_{2}\right)$.

(ii) $G_{b}$ has a $\left(\frac{d(d+1)}{2}, \frac{d(d+1)}{2}\right)$-tight edge-complete tower.

(B) If $q \in(1,2) \cup(2, \infty)$ then the following statements are equivalent.

(i) $G$ is essentially minimally rigid in $\left(\mathbb{R}^{d},\|\cdot\|_{q}\right)$.

(ii) $G_{b}$ has $a(d, d)$-tight edge-complete tower.

Proof (i) $\Rightarrow$ (ii) If $G$ is essentially minimally rigid in $\left(\mathbb{R}^{d},\|\cdot\|_{q}\right)$ then by Theorem $1.2, G_{b}$ contains a $(k, k)$-tight vertex-complete tower $\left\{G_{k}: k \in \mathbb{N}\right\}$ and this tower must be edge-complete. 
(ii) $\Rightarrow$ (i) If $G_{b}$ contains a $(k, k)$-tight edge-complete tower $\left\{G_{k, b}: k \in \mathbb{N}\right\}$ then by Theorem 1.2, $G$ is rigid. Let $v w \in E(G)$ be an inter-body edge and suppose $G \backslash\{v w\}$ is rigid. By Theorem 5.11, $G \backslash\{v w\}$ is sequentially rigid and so there exists a vertexcomplete tower $\left\{H_{k}: k \in \mathbb{N}\right\}$ in $G \backslash\{v w\}$ consisting of rigid subgraphs. Moreover, we can assume that each $H_{k}$ is a multi-body graph. Choose a sufficiently large $k$ such that $v, w \in V\left(H_{k}\right)$ and choose a sufficiently large $n$ such that $v w \in E\left(G_{n}\right)$ and $H_{k}$ is a subgraph of $G_{n}$. Then the body-bar graph for $H_{k} \cup\{v w\}$ is a subgraph of $\left(G_{n}\right)_{b}$ which fails the sparsity count for $\left(G_{n}\right)_{b}$. We conclude that $G \backslash\{v w\}$ is not rigid in $\left(\mathbb{R}^{d},\|\cdot\|_{q}\right)$ for all $v w \in E(G)$.

Open Access This article is distributed under the terms of the Creative Commons Attribution 4.0 International License (http://creativecommons.org/licenses/by/4.0/), which permits unrestricted use, distribution, and reproduction in any medium, provided you give appropriate credit to the original author(s) and the source, provide a link to the Creative Commons license, and indicate if changes were made.

\section{References}

1. Alexandrov, A.D.: Convex Polyhedra. Springer Monographs in Mathematics. Springer, Berlin (2005)

2. Asimow, L., Roth, B.: The rigidity of graphs. Trans. Am. Math. Soc. 245, 279-289 (1978)

3. Asimow, L., Roth, B.: The rigidity of graphs. II. J. Math. Anal. Appl. 68(1), 171-190 (1979)

4. Borcea, C.S., Streinu, I.: Periodic frameworks and flexibility. Proc. R. Soc. Lond. Ser. A, Math. Phys. Eng. Sci. 466(2121), 2633-2649 (2010)

5. Borcea, C.S., Streinu, I.: Minimally rigid periodic graphs. Bull. Lond. Math. Soc. 43(6), 1093-1103 (2011)

6. Bruhn, H., Diestel, R., Krieswell, M., Pendavingh, R., Wollan, P.: Axioms for infinite matroids. Adv. Math. 239, 18-46 (2013)

7. Cheng, J., Sitharam, M.: Maxwell-independence: a new rank estimate for the 3-dimensional generic rigidity matroid. J. Comb. Theory, Ser. B 105, 26-43 (2014)

8. Connelly, R., Shen, J.D., Smith, A.D.: Ball packings with periodic constraints. Discrete Comput. Geom. 52(4), 754-779 (2014)

9. Fogelsanger, A.L.: The Generic Rigidity of Minimal Cycles. PhD Thesis, Cornell University (1988)

10. Gluck, H.: Almost all simply connected closed surfaces are rigid. In: Glaser, L.C., Rushing, T.B. (eds.) Geometric Topology. Lecture Notes in Mathematics, vol. 438, pp. 225-239. Springer, Berlin (1975)

11. Gortler, S.J., Healy, A.D., Thurston, D.P.: Characterizing generic global rigidity. Am. J. Math. 132(4), 897-939 (2010)

12. Graver, J.E.: Counting on frameworks: Mathematics to Aid the Design of Rigid Structures. The Dolciani Mathematical Expositions, vol. 25. Mathematical Association of America, Washington, DC (2001)

13. Graver, J., Servatius, B., Servatius, H.: Combinatorial Rigidity. Graduate Texts in Mathematics, vol. 2. American Mathematical Society, Providence (1993)

14. Jackson, B., Jordán, T.: Connected rigidity matroids and unique realizations of graphs. J. Comb. Theory, Ser. B 94(1), 1-29 (2005)

15. Jackson, B., Jordán, T., Szabadka, Z.: Globally linked pairs of vertices in rigid frameworks. In: Connelly, R., et al. (eds.) Rigidity and Symmetry. Fields Institute for Research in Mathematical Sciences, vol. 70, pp. 177-203. Springer, New York (2014)

16. Kann, E.: Infinitesimal rigidity of almost-convex oriented polyhedra of arbitrary Euler characteristic. Pac. J. Math. 144(1), 71-103 (1990)

17. Kastis, E., Power, S.C.: The first-order flexibility of a crystal framework (2018). arXiv:1802.00980

18. Katoh, N., Tanigawa, S-i: A proof of the molecular conjecture. Discrete Comput. Geom. 45(4), 647-700 (2011)

19. Kitson, D.: Finite and infinitesimal rigidity with polyhedral norms. Discrete Comput. Geom. 54(2), 390-411 (2015) 
20. Kitson, D., Power, S.C.: Infinitesimal rigidity for non-Euclidean bar-joint frameworks. Bull. Lond. Math. Soc. 46(4), 685-697 (2014)

21. Kitson, D., Power, S.C.: The rigidity of infinite graphs II (preprint)

22. Kitson, D., Schulze, B.: Maxwell-Laman counts for bar-joint frameworks in normed spaces. Linear Algebra Appl. 481, 313-329 (2015)

23. Laman, G.: On graphs and the rigidity of plane skeletal structures. J. Eng. Math. 4(4), 331-340 (1970)

24. Malestein, J., Theran, L.: Generic combinatorial rigidity of periodic frameworks. Adv. Math. 233, 291-331 (2013)

25. Maxwell, J.C.: On the calculation of the equilibrium and stiffness of frames. Philos. Mag. 27, 294-299 (1864)

26. Nash-Williams, C.St.J.A.: Decomposition of finite graphs into forests. J. Lond. Math. Soc. 39(1), 12 (1964)

27. Nash-Williams, C.St.J.A.: Infinite graphs—a survey. J. Comb. Theory 3, 286-301 (1967)

28. Nixon, A., Owen, J.C., Power, S.C.: Rigidity of frameworks supported on surfaces. SIAM J. Discrete Math. 26(4), 1733-1757 (2012)

29. Nixon, A., Owen, J.C., Power, S.C.: A characterization of generically rigid frameworks on surfaces of revolution. SIAM J. Discrete Math. 28(4), 2008-2028 (2014)

30. Owen, J.C., Power, S.C.: Infinite bar-joint frameworks. In: Proceedings of the 2009 ACM Symposium on Applied Computing (SAC'09), pp. 1116-1121. ACM, New York (2009)

31. Owen, J., Power, S.: Continuous curves from infinite Kempe linkages. Bull. Lond. Math. Soc. 41(6), 1105-1111 (2009)

32. Owen, J.C., Power, S.C.: Infinite bar-joint frameworks, crystals and operator theory. New York J. Math. 17, 445-490 (2011)

33. Oxley, J.: Infinite matroids. In: White, N. (ed.) Matroid Applications. Encyclopedia of Mathematics and Its Applications, vol. 40, pp. 73-90. Cambridge University Press, Cambridge (1992)

34. Power, S.C.: Polynomials for crystal frameworks and the rigid unit mode spectrum. Philos. Trans. R. Soc. Lond., Ser. A, Math. Phys. Eng. Sci. 372(2008), 20120030 (2014)

35. Power, S.C.: Elementary proofs of Kempe universality. Math. Proc. R. Ir. Acad. 117A(1), 23-37 (2017)

36. Ross, E.: The rigidity of periodic body-bar frameworks on the three-dimensional fixed torus. Philos. Trans. R. Soc. Lond., Ser. A, Math. Phys. Eng. Sci. 372(2008), 20120112 (2014)

37. Ross, E., Schulze, B., Whiteley, W.: Finite motions from periodic frameworks with added symmetry. Int. J. Solids Struct. 48(11-12), 1711-1729 (2011)

38. Schulze, B.: Symmetric versions of Laman's theorem. Discrete Comput. Geom. 44(4), 946-972 (2010)

39. Sitharam, M., Willoughby, J.: On flattenability of graphs. In: Botana, F., Quaresma, P. (eds.) Automated Deduction in Geometry. Lecture Notes in Artificial Intelligence, vol. 9201, pp. 129-148. Springer, Cham (2015)

40. Tay, T.-S.: Rigidity of multigraphs I. J. Combin. Theory Ser. B 36(1), 95-112 (1984)

41. Wegner, F.: Rigid-unit modes in tetrahedral crystals. J. Phys. Condens. Matter 19(40), 406218 (2007)

42. Whiteley, W.: Infinitesimally rigid polyhedra I: Statics of frameworks. Trans. Amer. Math. Soc. 285(2), 431-465 (1984)

43. Whiteley, W.: Infinitesimally rigid polyhedra. II: Modified spherical frameworks. Trans. Amer. Math. Soc. 306(1), 115-139 (1988)

44. Whiteley, W.: The union of matroids and the rigidity of frameworks. SIAM J. Discrete Math. 1(2), 237-255 (1988)

45. Whiteley, W.: Matroids and rigid structures. In: white, N. (ed.) Matroid Applications. Encyclopedia of Mathematics and its Applications, vol. 40, pp. 1-53. Cambridge University Press, Cambridge (1992) 